\title{
Grundzüge des deutschen Krankenhauswesens von 1780 bis 1930 unter Berücksichtigung von Schweizer Vorbildern
}

\author{
Von Axel Hinrich Murken
}

Krankenhaus und Krankenpflege, Klinik und klinische Medizin sind in unserer abendländischen Gesellschaft so selbstverständliche Begriffe geworden, daß wir kaum noch über ihre Ursprünge und über die lange, bis ins Mittelalter reichende Entwicklungsgeschichte nachdenken. Jeder Bürger kommt in Westeuropa, wenn man den Statistiken glauben darf, ein- bis zweimal in seinem Leben wegen Gesundheitsstörungen, oder weil sein Leben zu Ende geht, mit dem Krankenhaus oder der Klinik in meistens großer existentieller Not in Berührung. Darüber hinaus werden ihm durch Rundfunk und Fernsehen, durch die Werbung, durch Zeitschriftenberichte oder durch die Trivialliteratur, in der der im Krankenhaus spielende Arztroman ein ganzes Genre bildet, bis ins Wohnzimmer hinein Eindrücke aus diesem Bereich unseres Alltags vermittelt. «Klinisch getestet» ist ein Schlagwort und Gütesiegel für fast alles geworden, was in irgendwelcher Form als Applikation vom Medikament bis zum Kosmetikum für das körperliche Wohlbefinden empfohlen wird. Man kann mit Recht sagen, daß ganze Industriezweige das Milieu der Klinik, das dortige Wirken von Ärzten und Krankenschwestern und die überlieferte passive Rolle des Patienten (lat. pati $=$ sich gefallen lassen, erdulden) mitformen und spiegeln.

Doch so neu ist das Krankenhaus, selbst das Mammutkrankenhaus der Gegenwart, wie es sich jüngst in Aachen, Münster oder Wien darstellt, nicht. Andererseits verfügt die heutige Heilkunde aufgrund der seit Mitte des 19. Jahrhunderts kaum erahnbaren Fortschritte der klinischen Medizin auf der Basis der exakten Naturwissenschaften tatsächlich über eine ungewöhnliche Leistungsfähigkeit und -bereitschaft, wie es nie zuvor in der Geschichte der Medizin der Fall war. Dazu hat ganz entscheidend das Krankenhauswesen beigetragen, da besonders in dem Bereich dieser sozial-karitativen Institution die moderne Heilkunde, die klinische Medizin, eigentlich erst entwickelt werden konnte.

Trotz dieser großen Erfolge der heutigen Heilkunde macht sich zugleich ein kaum noch zu übersehender Skeptizismus gegenüber der Medizin breit, 
der sich besonders gegen die klinische Medizin und ihre Institutionen richtet. Man mißtraut den modernen Mammutkliniken, die in den prosperierenden goldenen sechziger und siebziger Jahren mit ungeheuren Finanzmitteln in den europäischen Städten entstanden sind. Ähnlich wie im 19. Jahrhundert, als man seit der Blüte der Biedermeierzeit vor dem Hintergrund einer fast ungestörten industriellen Entwicklung in den größeren städtischen Zentren Mitteleuropas, wie in Basel, Hamburg, München, Stuttgart, Wien oder Zürich, für die damalige Medizin vorbildliche Krankenhäuser baute, sind Angst und Spott des Bürgers vor diesen Anstalten, in denen man sich seelenloser Medizintechnik ausgeliefert zu sein glaubt, kaum noch zu übersehen. Damals kamen gegenüber den neuen Krankenhausbauten teilweise disqualifizierende Schlagworte wie «Armenpaläste», «Seuchenasyle» oder «Luxusherbergen» auf, denen man heute Bezeichnungen wie «Gesundheitsfabriken», «Hoffnungsburgen», «TÜV-Stationen» oder (analog zu den Risiken der Raumfahrt) «Cap Canaverals» zur Seite stellen kann. Kein Geringerer als Rudolf Virchow (1821-1902) äußerte sich schon 1849 in der «Medizinischen Reform» - fast beispielhaft für seine Generation - sehr abfällig über den spektakulären Neubau der 1847 vollendeten Berliner Diakonissenanstalt Bethanien, eines der großzügigsten Krankenhäuser der Biedermeierzeit. Er scheute sich nicht, deshalb den Preußenkönig Friedrich Wilhelm IV., der das Geld dazu gegeben hatte, öffentlich anzugreifen ${ }^{1}$.

Auch andere bedeutende Ärzte der Gründerzeit wie Theodor Billroth (1829-1894) standen dem Krankenhauswesen sehr skeptisch, wenn nicht sogar negativ gegenüber. Vor dem Hintergrund der heutigen Diskussion um das Krankenhauswesen bekommt die Geschichte dieser Anstalten mit all ihren Verflechtungen in die allgemeine Kultur- und Sozialgeschichte eine besonders aktuelle Bedeutung. Man sollte sich immer wieder in Erinnerung rufen, daß sehr unterschiedliche Modelle von Hospitälern und Krankenhäusern seit dem Mittelalter verwirklicht worden sind, die besonders in den letzten 200 Jahren mit ihren positiven und negativen Seiten nicht nur bei den Experten, sondern auch in der Bevölkerung ein breites Echo gefunden haben. Dafür liefern uns vor allem die zahlreichen Berichte und Bilder in den Familienzeitschriften des vorigen Jahrhunderts (Gartenlaube, Leipziger Illustrirte Zeitung) wertvolle Dokumente. Die Palette der für uns lehrreichen Beispiele reicht vom Großkrankenhaus mit 1000 bis 2000 Krankenbetten, das schon im Paris des 18. Jahrhunderts eine große Bedeutung hatte, bis zur Zwergklinik mit wenigen Unterbringungsmöglichkeiten für stationär zu versorgende Patienten. Und in den letzten 100 Jahren erleben wir ein 
Pendeln der Planer zwischen dem Hochhauskrankenhaus und der eingeschossigen Lazarettbaracke, zwischen zentralen und dezentralen Anstaltsbauten. Das Für und Wider um die Integration psychisch Kranker in allgemeine Krankenanstalten gehört seit der frühen Biedermeierzeit ebenso dazu wie die vielzähligen Tageskliniken, die seit 1840 für die Betreuung von kranken Kindern, verkrüppelten Individuen, unehelichen Schwangeren und Augenkranken in vielen Städten des deutschsprachigen Raumes aus dem Boden schossen.

Gleichfalls hat die Abtrennung von Infektionskrankheiten oder von ansteckungsverdächtigen Personen eine lange Tradition. Schon im Mittelalter findet man unterschiedliche Absonderungshäuser für Lepra-, Pest- und schließlich seit 1500 für Syphiliskranke. Im vorigen Jahrhundert richtete man angesichts der gefürchteten Pocken- und Choleraepidemien, der verheerenden Tuberkulose und der Diphtherie, gegen die man lange Zeit keine auch nur einigermaßen erfolgversprechende Therapie hatte, Isolierhäuser, Barackenlazarette oder streng bewachte Absonderungsgebäude ein.

Die christliche Barmherzigkeit:

Wegbereiterin des Hospital- und Krankenhauswesens

Wenn man die moderne Krankenhausmedizin verstehen will, die an sich unmittelbar mit der Geburt der Klinik am Vorabend der Französischen Revolution von 1789 verknüpft ist, muß man kurz an den wesentlichen Ursprung des Hospitals und des Krankenhauses erinnern: der christlichen Barmherzigkeit. Mit der Einbeziehung der Krankenpflege in die sechs Werke der Barmherzigkeit, die uns der Evangelist Matthäus überliefert, war der Boden für eine unvergleichlich karitative Tätigkeit bereitet, die die Antike in all ihren kulturellen Schat tierungen nicht gekannt hat und die weit über den antiken Humanitätsbegriff hinausgeht. Der christliche Gedanke, daß man im Kranken Christus selbst pflegt, schuf schließlich die Grundlage für die Bildung von Ordensgemeinschaften, die wie beispielhaft die Benediktiner in ihren Regeln der Pflege der Kranken breiten Raum gaben: Infirmorum cura ante omnia et super omnia adhibenda est!

Der berühmte bauliche Entwurf für das Benediktinerkloster St. Gallen aus dem Jahre 820, der allerdings in seiner großartigen, übersichtlichen Konzeption utopisch blieb, sah nicht nur ein großes Areal des Klosterbezirkes, das sogenannte «Infirmarium», für die erkrankten Ordensbrüder vor, 
sondern verfügte auch am Tor des Klosters über ein Pilgerhospiz und eine bescheidene Fremdenherberge, die für die peregrini et pauperes gedacht waren. Diese zweigleisige bauliche Struktur für die Beherbergung der Pilger je nach Rang, Stand und Befinden läßt sich in den späteren Klosteranlagen der Benediktiner immer wieder finden (z. B. Fulda, Osnabrück, Reichenau, St. Gallen). Die bald den Klöstern angegliederten Spitäler im unmittelbaren Bereich der Klosterpforte bildeten die Vorstufe zu den städtischen Hospitälern des hohen Mittelalters. So wurden seit dem 14. Jahrhundert die mittelalterlichen Hospitäler, die ursprünglich in enger Kommunikation mit den Bischofssitzen oder mit Ordensklöstern der Benediktiner oder Franziskaner entstanden, als eigenständige Institutionen immer mehr in das Straßenbild der Städte integriert. Dies war seit dem Beginn des 13. Jahrhunderts vor allem das Verdienst von ordensähnlich organisierten Pflegegemeinschaften, die wie der Orden der Brüder vom Heiligen Geist oder wie die

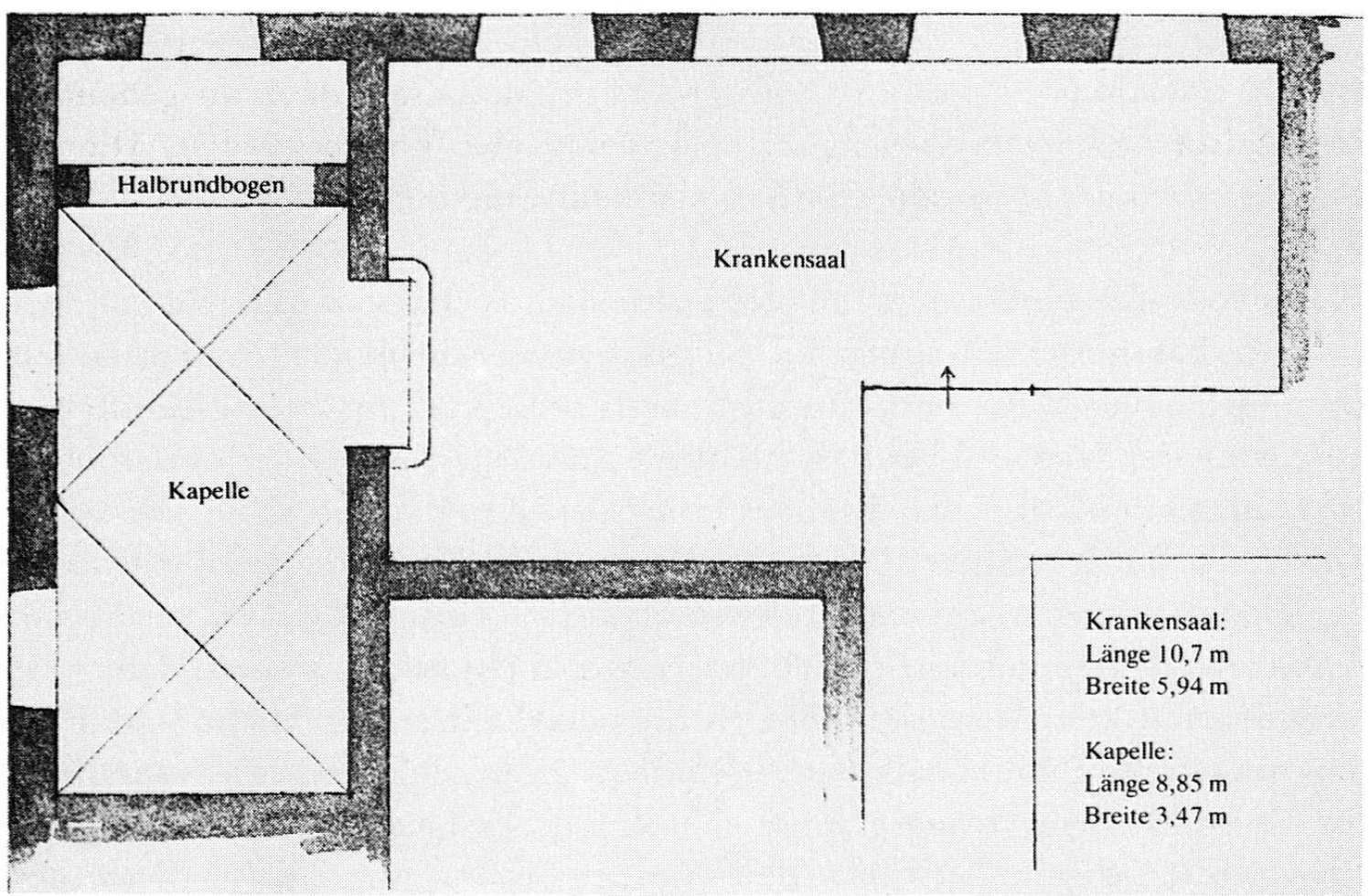

Abb. 1. Grundriß des Spitaltraktes auf der Burg des Deutschen Ordens in Sumiswald aus dem 13. Jahrhundert. Die unmittelbare Verknüpfung der querliegenden Kapelle mit dem Krankensaal ist charakteristisch für Spitalsanlagen im hohen Mittelalter. Nach einer Zeichnung von Alex Walter Diggelmann in: Marta Meyer-Salzmann, Geschichte der Medizin im Emmental, Sumiswald 1979, S.27 
Deutschordensritter eine Vielzahl von Hospitälern unter ihrem Namen in der Schweiz und in Deutschland betrieben. Eines der schönsten Spitäler des Deutschen Ordens, das heute noch erhalten ist, steht - verbunden mit einer Burganlage - in Sumiswald im schweizerischen Emmental ${ }^{2}$ (Abb. 1).

Seit dem Herbst des Mittelalters versuchten aber auch die Kommunen im Zusammenhang mit ihrer allmählichen Abgrenzung gegenüber den Territorialherren - nicht zuletzt mittels der seit dem 14. Jahrhundert immer häufiger nachweisbaren Stadtärzte - nach und nach den Einfluß auf das Hospitalwesen in ihre Mauern zu bekommen. Allerdings überließen sie, ebenso wie die Landesfürsten, den Unterhalt dieser Wohlfahrtseinrichtungen gern den religiösen Genossenschaften oder bürgerlichen Stiftungen. Einer der führenden Hospitalorden war der Heilig-Geist-Orden, aber auch die Alexianer, Dominikaner und schließlich die Barmherzigen Brüder und die Elisabethinerinnen widmeten sich seit dem 16. bzw. 17. Jahrhundert ganz gezielt neben der offenen (häuslichen) auch der geschlossenen Krankenpflege.

Doch auch in den Gasthäusern, die von Pächtern nach privatwirtschaftlichen Grundsätzen betrieben wurden, hatte man seit dem ausgehenden Mittelalter Krankenstuben für nicht einheimische Wandergesellen, Dienstboten und Handelsreisende im Krankheitsfall eingerichtet. Hier kehrten oft auch umherfahrende Steinschneider und Okulisten ein (Abb.2). Häufig verfügten die größeren Stadtgemeinden schon seit dem Spätmittelalter über ein sehr differenziertes, geschlossenes Anstaltswesen für die Versorgung der gebrechlichen Armen und Kranken. So hatte Luzern im 16. Jahrhundert neben dem Heiliggeist-Hospital, dem «ersten und fürnembsten houptspittal», ein Leprosorium, ein Pesthaus, ein Spital für die «Blattersüchtigen» und eines, das den mit der «Krankheit der franzosen beschwärten Lüten» zugedacht war. Nicht weniger als fünf verschiedene Hospitaltypen sind demnach in Luzern in der Renaissancezeit nachweisbar ${ }^{3}$.

Seelische Ansprache und körperliche Versorgung hielten sich, soweit es die praktische Medizin damals überhaupt vermochte, im Hospital der religiösen Ordensgemeinschaften die Waage. Wenn auch die geistliche Betreuung, die seelische Reinigung von den Sünden am Beginn der Aufnahme in das Hospital standen und baulich durch die Einbeziehung eines Altars berücksichtigt wurden, so war doch der offiziell niedergelassene Stadtarzt oder Wundchirurg kein Unbekannter im Krankensaal. Die ersten offiziellen Stadtärzte sind in Westeuropa seit dem 13. Jahrhundert ausfindig zu machen (z. B. Luzern 1296, Aachen 1346). Das Basler Leonhards-Spital 


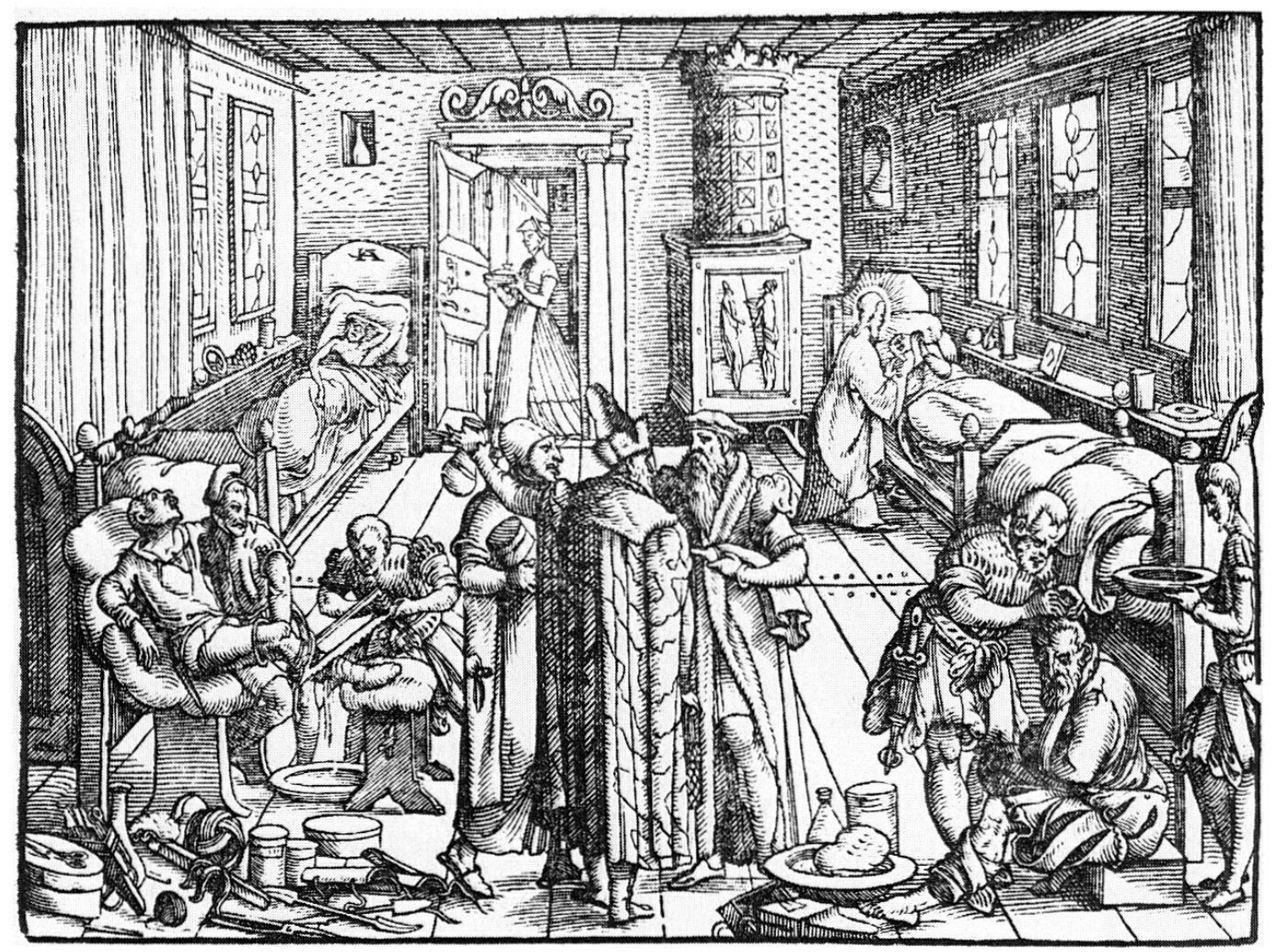

Abb.2. Blick in eine Krankenstube der Renaissance im deutschsprachigen Gebiet. Vermutlich handelt es sich um einen für Kranke eingerichteten Raum, der einem Gasthaus zugeordnet war. Links und rechts erkennt man Wundärzte in chirurgischer Tätigkeit. In der Mitte disputieren zwei Ärzte bei der Harnschau. Ein Altar fehlt; jedoch kann man in dem Krankenpfleger rechts am Bett (Strahlenkrone, Barttracht, Gewand) eine christusähnliche Gestalt erblicken. Holzschnitt aus: Paracelsus, Opus Chyrurgicum, Frankfurt am Main 1566, S. 148. Herzog-August-Bibliothek, Wolfenbüttel

verfügte seit Beginn des 14. Jahrhunderts sogar über einen chirurgisch versierten Spitalmeister. Sofern man nicht, wie die Barmherzigen Brüder (1540 in Granada gegründet), die im 17. Jahrhundert nördlich der Alpen in Mitteleuropa Fuß faßten (Neuburg an der Donau 1622), in den eigenen Reihen in der Medizin und Pharmazie erfahrene Mitglieder ausgebildet hatte, verpflichtete man auch in den Ordensspitälern die ansässigen Stadtoder Leibärzte.

Im 18. Jahrhundert, dem Zeitalter der Aufklärung, genügte jedoch das aus dem Mittelalter überkommene Hospital mit seinen teilweise überalterten Strukturen nicht mehr den gesellschaftlichen und gesundheitspolitischen Anforderungen eines aufgeklärten Absolutismus. Die großen, zwan- 
zig, dreißig oder mehr Betten umfassenden Hospitalsäle erlaubten vor allem keine soziale Differenzierung der Patienten. Noch wesentlicher aber war,

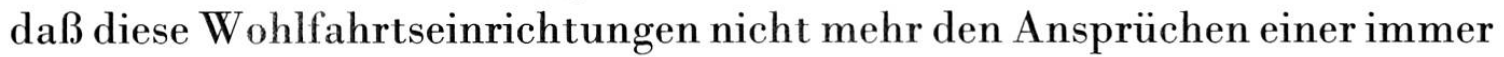
mehr von den Naturwissenschaften beeinflußten Medizin gerecht wurden. Neuere hygienische und gesundheitspolitische Gesichtspunkte konnten häufig in den alten Mauern der Hospitäler, die inzwischen trotz der seit Mitte des 18. Jahrhunderts beginnenden Stadterweiterungen in enge Wohnviertel eingezwängt worden waren, nicht berücksichtigt werden. Viele Hospitäler degenerierten gleichsam zu reinen Pfründneranstalten oder zu Asylen von obdachlosen, ärmlichen, erbarmungswürdigen menschlichen Existenzen. Für die vom Land hinzugezogenen Handwerksgesellen, Dienstboten und Arbeiter fehlten jetzt die entsprechenden karitativen Einrichtungen im Krankheitsfall. Sie mußten häufig in den Gasthöfen versorgt werden.

Eine ähnliche Entwicklung hatte sich schon im 16. und 17. Jahrhundert für die Absonderungsspitäler abgezeichnet. In den seit dem 10. Jahrhundert jenseits der Stadtwälle nachweisbaren Isolierhäusern für Seuchenkranke verwahrte man seit der Renaissance häufig neben Leprösen, Pest- oder Pockenkranken auch sonstige Infektionsverdächtige (z.B.Syphilitiker, Ignis-sacer-Kranke). Unter den Seuchenhäusern kann man besonders zwei Typen klassifizieren: die Leprosorien (Gutsleuthäuser, Melaten) und die Pesthäuser (Lazarette, Blatternhäuser). Während die Lazarette streng hermetisch, häufig durch zusätzliche Wassergräben abgeschlossen waren (z. B. Pesthaus in Leiden), lagen die Leprosorien an den Hauptstraßen, wo eine Kommunikation mit den Leprakranken leicht möglich war (Abb.3).

Später, im 18. Jahrhundert, nachdem vor allem die Pestepidemien ihren großen Schrecken verloren hatten, brachte man auch andere, als asozial eingeschätzte Individuen (Heimatlose, Sieche, Irre, chronisch Kranke ohne Angehörige) in diesen Häusern unter, die man aus dem Weichbild der Städte entfernt haben wollte. Teilweise entwickelten sich aber aus diesen Seuchenasylen, wie aus dem «Prestenhaus» in St.Gallen, den Pesthäusern von Hamburg oder Berlin oder den Lazaretten in Dresden oder Hannover, die ersten Vorstufen moderner Krankenanstalten (Abb.4).

\section{Die ersten Staatskrankenhäuser des 18.Jahrhunderts}

In Deutschland wurde die entscheidende Wende in der Reformierung des Hospital- und Krankenhauswesens durch die Gründung der Charité in 


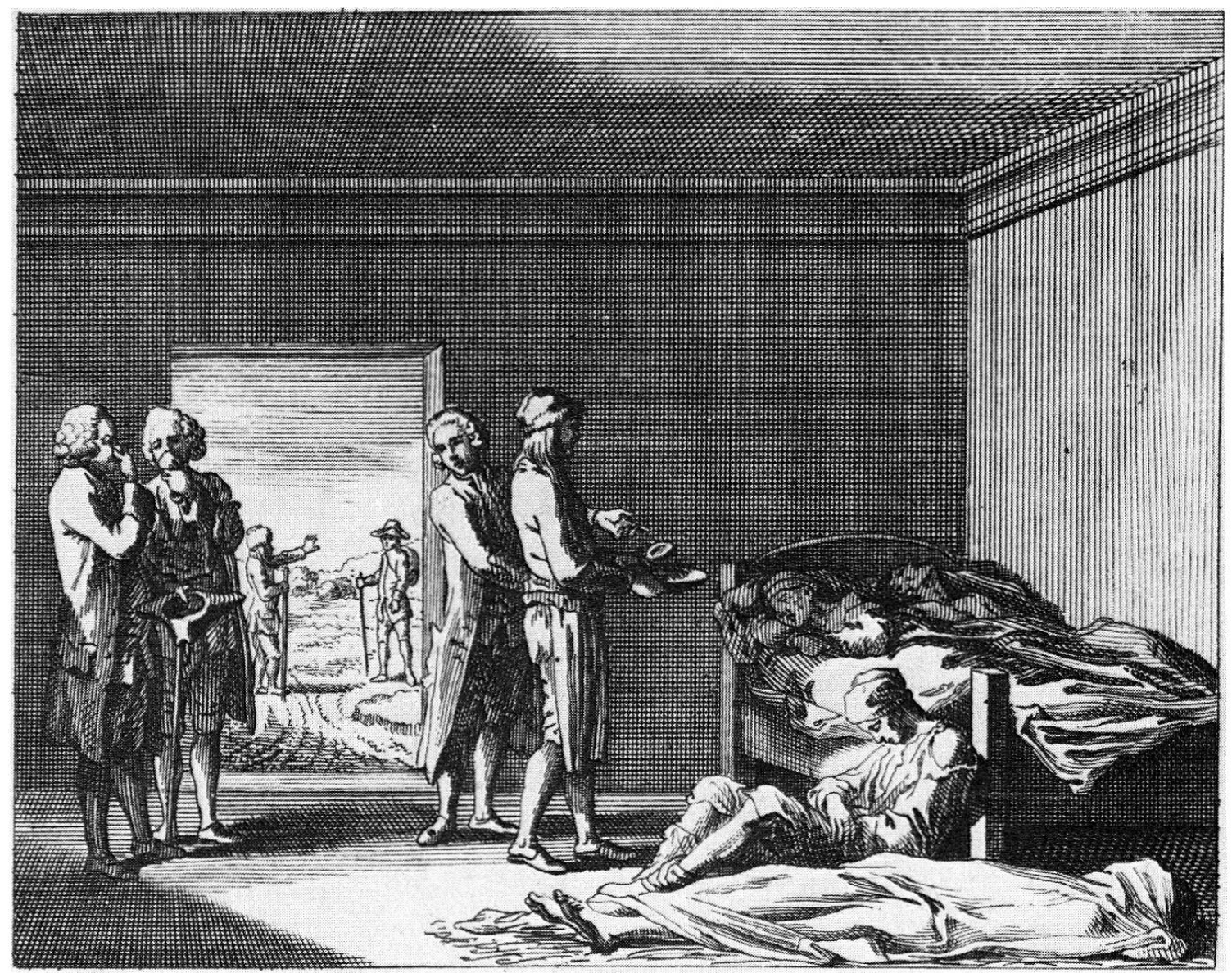

Abb.3. Charakteristische Darstellung eines Krankensaals in einem Pesthaus mit Blick nach draußen. Im Bett liegen zwei Kranke, die von zwei Wärtern versorgt werden. Vorn rechts und vor dem Bett ein Toter und ein Sterbender. Links halten sich zwei Ärzte «Filtermasken», wahrscheinlich mit Essig getränkte, mehrfach gefaltete Tücher, vor Mund und Nase. Die offene Eingangstür zeigt einen Blick in die freie Natur; ein bewaffneter Soldat weist einen Besucher ab. Radierung von Johann F. Schuster (1666-1724), Krankenstube eines Lazarettes. 18. Jahrhundert. Kunstsammlungen Veste Coburg

Berlin 1727 in dem 1710 vollendeten, nicht benutzten Pesthaus eingeleitet (Abb.5). Unter den Preußenkönigen Friedrich Wilhelm I. und Friedrich II. sollte die Charité bald immer mehr europäisches Gewicht gewinnen. Wahrscheinlich lieferten Londoner Krankenhäuser, wie das 1724 vollendete Bartholomew's Hospital, und Pariser Hospitäler, wie das berühmte Hôpital de la Charité (1608), und nicht zuletzt der Neubau des Inselspitals in Bern (1718-1724), in dem neben den Pfründnern 40 Kranke stationär versorgt werden konnten, die wirksamsten Vorbilder zu diesem ersten großen Staatskrankenhaus in Preußen. Man muß aber auch erwähnen, daß einige 
Städte schon wesentlich früher Spitäler (Nosokomien) nur für heilbare Kranke verwendet wissen wollten oder neu eingerichtet hatten (Bern 1531, Bremen 1697). Die Bezeichnung Krankenhaus, die der Enzyklopädist Johann Georg Krünitz (1728-1796) 1789 als sinnvoll empfahl, bürgerte sich erst später ein.

\section{Iffertumalicifi Erinowitum

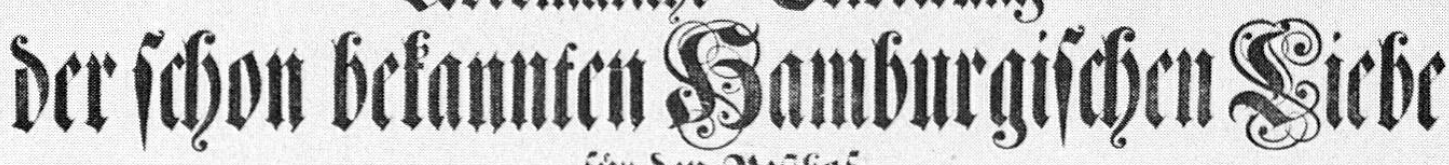

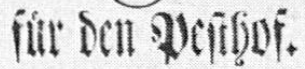

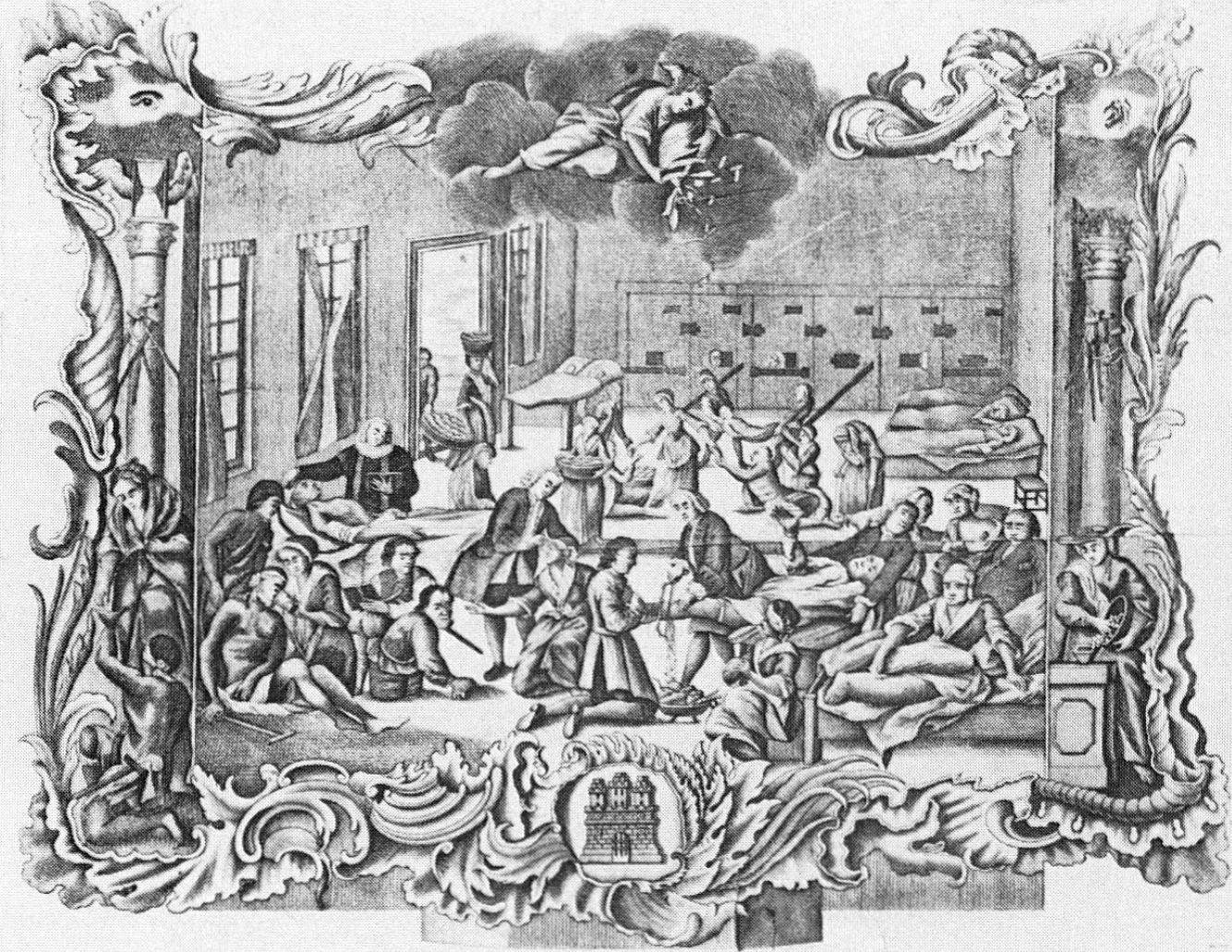

Abb.4. Blick in einen großen Krankensaal des seit 1606 nachweisbaren Pesthofes in Hamburg, der seit 1742 auf den jährlichen Bittbriefen der Freien Hansestadt immer wieder dargestellt wird. Im Saal werden verschiedene chirurgische Eingriffe durchgeführt; links tröstet ein Geistlicher einen Sterbenden. Im Hintergrund zweischläferige Krankenbetten vor gefängnisartigen Türen; durch zwei geöffnete Eßluken stecken Geisteskranke ihr Köpfe. Seit 1797 bezeichnete man diese Anstalt als «Krankenhof». Kupferstich von Philipp Andreas Kilian (1714-1759) nach einem Gemälde von F.S. Heintze. Nachdruck aus dem Jahre 1782 mit dem Aufruf zur Spende durch den Provisor Detlev Nordhoff. Privatbesitz Aachen 
Man errichtete in der preußischen Metropole in dem an sich für Pestkranke 1710 bestimmten vierflügeligen Baukomplex unter anderem eine Krankenanstalt mit 200 Betten (vgl. Abb.5). Aber diese Anstalt war noch mit einer Pfründneranstalt verbunden, wie es auch bei den großen deutschen Renaissance-Spitälern (Würzburg: Juliusspital 1576, München: ElisabethSpital 1624) der Fall gewesen war. Ebenso fanden noch ein Militärlazarett und ein Wöchnerinnenasyl für «liederliche Weibspersonen» in dem vierflügeligen Gebäudekomplex nicht weit vor dem Berliner Brandenburger Tor ihren Platz. Zugleich diente dieses Haus mit seinen sechs Fachabteilungen als Ausbildungsstätte für Wundärzte des preußischen Heeres.

Charakteristisch für den aufgeklärten Absolutismus war, daß man die ärztliche Leitung einer Dreierdirektion übertragen hatte, wobei der Internist Johann Theodor Eller (1689-1760) durch sein wissenschaftliches Werk besonders herausragte. Die Krankenzimmer mit 12 bis 18 und mehr Betten waren sehr eng und häufig überfüllt (Abb.6). Aber jeder Kranke hatte
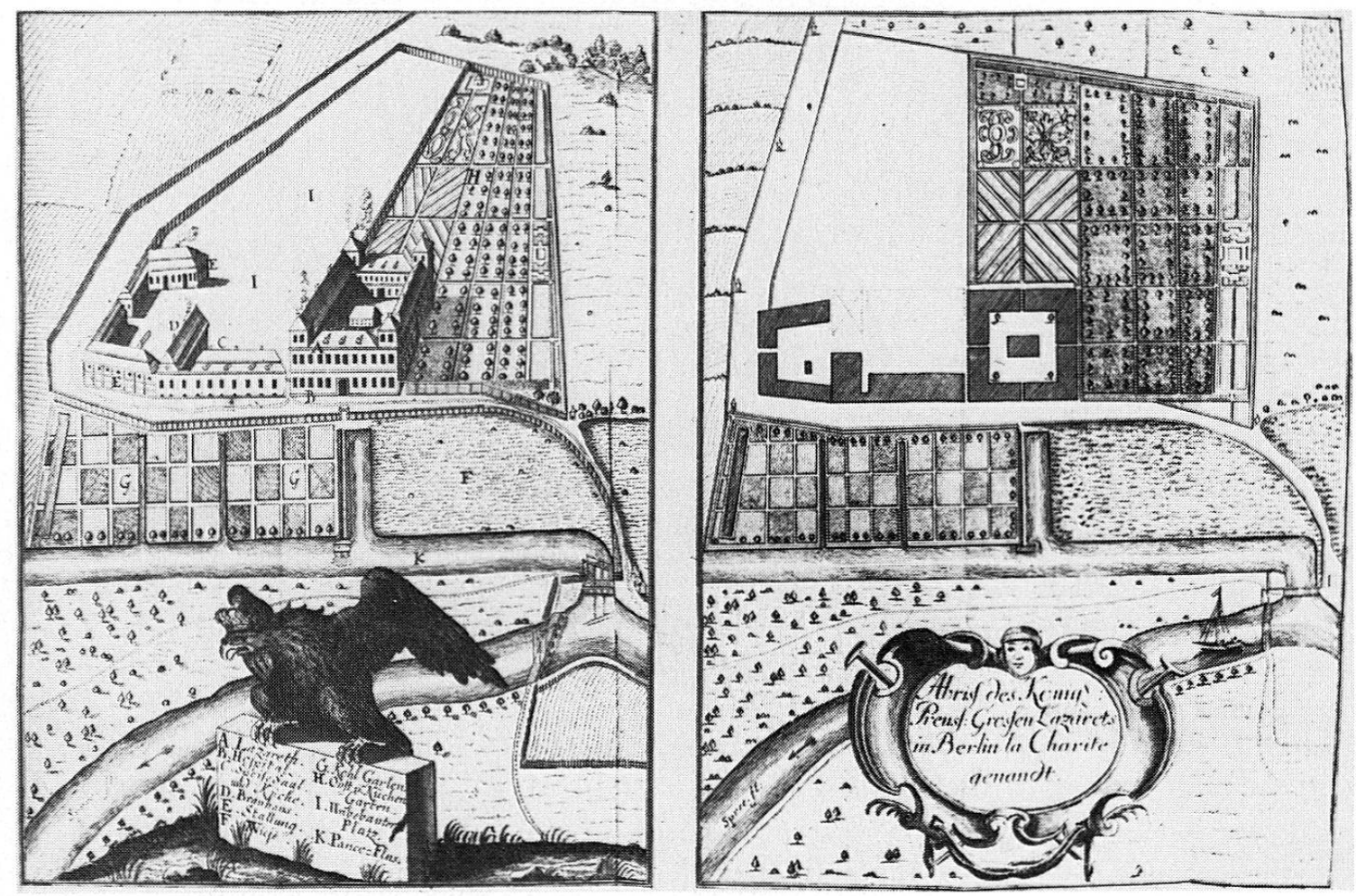

Abb. 5. Ansicht aus der Vogelschau und Situationsplan von der Charité in Berlin. Unbezeichneter Kupferstich aus: Johann Theodor Eller, Nützliche und auserlesene medicinische und chirurgische Anmerckungen ..., Berlin 1730, nach S. 14. Universitätsbibliothek Münster 


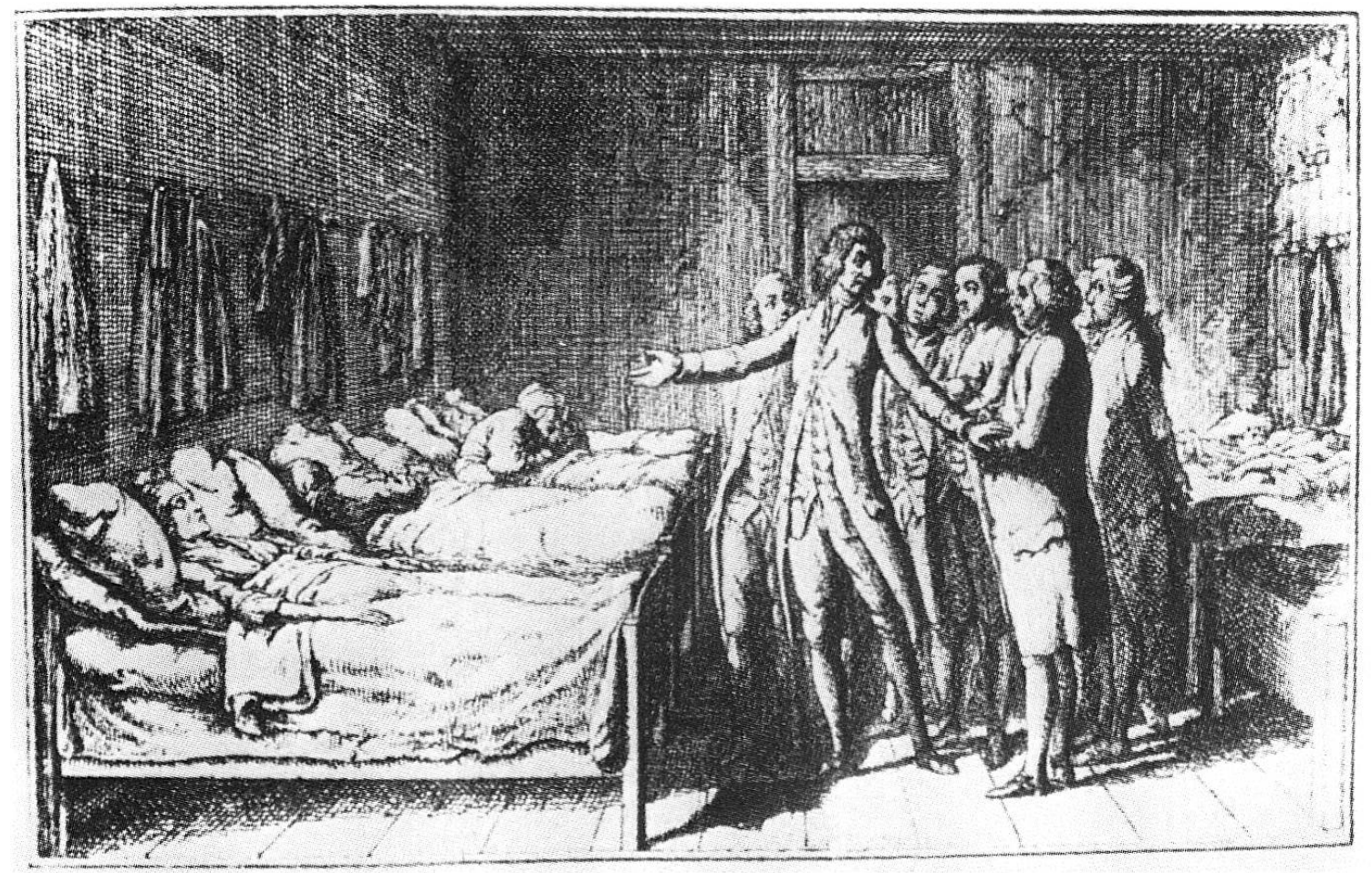

Abb.6. Blick in einen Krankensaal der Berliner Charité (?) während der morgendlichen Ärztevisite. Kupferstich nach einer Zeichnung von Daniel Chodowiecki (1726-1801). Dieser interessante kleine Kupferstich $(68 \times 115 \mathrm{~mm})$ ist einer Erzählung von Matthias Claudius, Der Besuch im St.Hiob zu ..., beigefügt, die im Wandsbecker Bothen 1783, 4.Teil, S.96, erschien. Privatbesitz Aachen

wenigstens sein eigenes Bett, was - abgesehen von den Hospitälern der Barmherzigen Brüder - durchaus nicht überall üblich war, eine Matratze, einen Strohsack und weiße, saubere Wäsche. Noch im Jahr der Einweihung baute man ein drittes Geschoß aus, in dem man einen Operationssaal mit Instrumentenschränken und sonstigen für Steinschnitte, für Bruch- und Fisteloperationen oder für Amputationen «gehörigen Geräteschaften» aufbewahrte. Die Verwaltung dieses großen Krankenhauses übertrug man einem Inspektor, der sehr sparsam zu wirtschaften hatte und den Ärzten in allem streng auf die Finger sah. Viele Kritiker dieses preußischen Staatskrankenhauses, unter ihnen der berühmte Charité-Chirurg Ernst Horn (1774-1848), sahen in dem mangelnden Einfluß der Ärzte auf die Administration die Ursache für die teilweise skandalösen hygienischen Verhältnisse, die sich bald hinter den Mauern der Charité breitmachten.

Bezeichnend für die Signalfunktion, die die Berliner Charité hatte, war, 
daß man wenige Jahre nach ihrer Eröffnung in Stettin (1733-1734), in Hannover (1734-1736) und später in Braunschweig (1764-1780) sogenannte bürgerliche Stadtlazarette oder Armenkrankenhäuser ins Leben rief (Abb.7). In diesen kleineren Krankenhäusern betreute in der Regel der Stadtphysikus oder der Leibarzt des Fürsten zweimal in der Woche die Kranken, die bei Vorlage des Armenscheins in den Genuß einer kostenlosen krankenpflegerischen, medikamentösen und ärztlichen Behandlung kamen. In einigen Städten des deutschsprachigen Gebietes kann man aber schon wesentlich früher, wie schon erwähnt, nur für den Krankenhausdienst angeworbene und honorierte Spitalärzte nachweisen. Meistens handelte es sich dabei um erfahrene Wundchirurgen. Sie unterstanden den dafür mitbesoldeten Stadtärzten. In der zweiten Hälfte des 18. Jahrhunderts rief man eine Reihe von weiteren Allgemeinen Krankenhäusern ins Leben, die

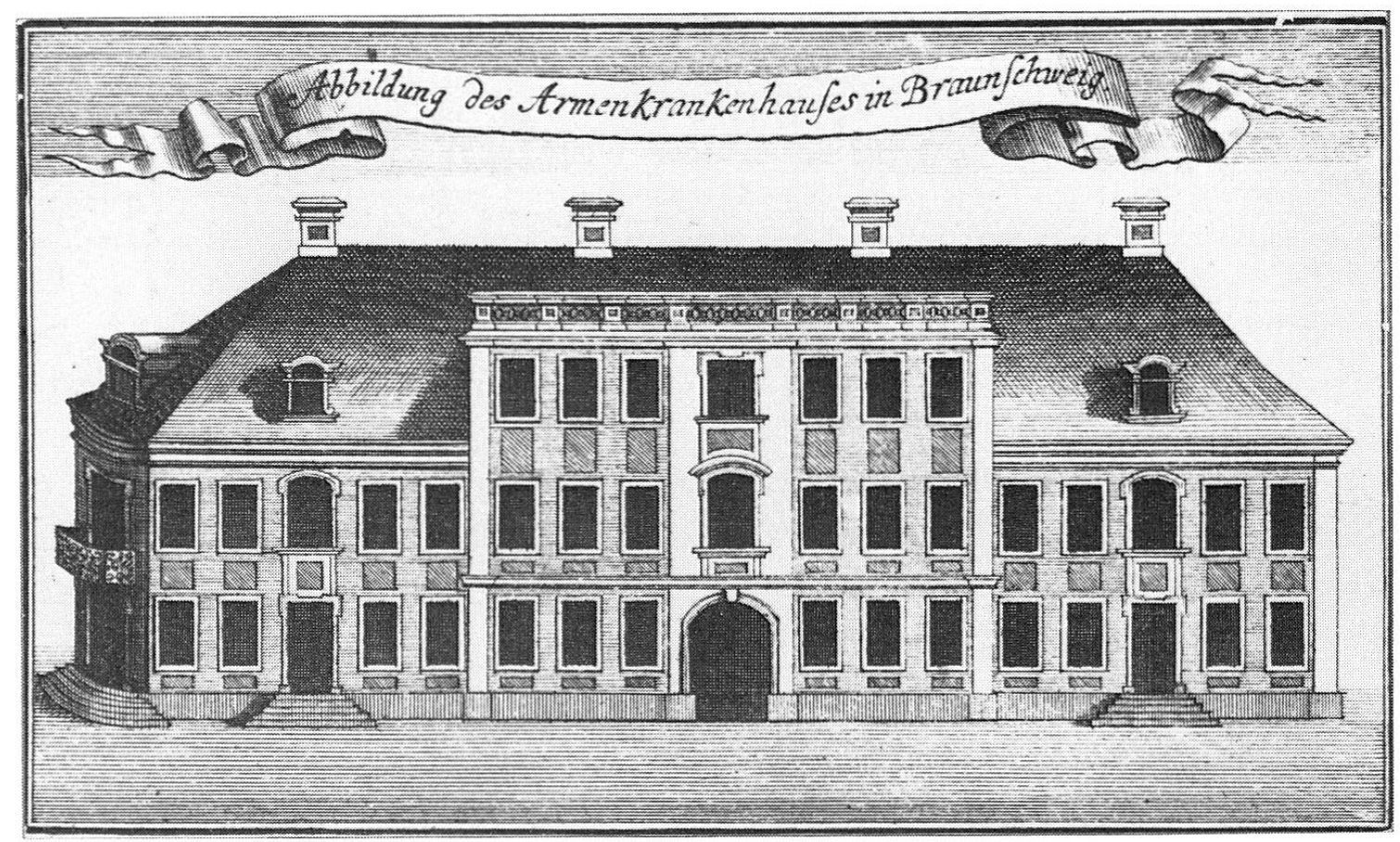

Abb.7. Ansicht vom Herzoglichen Armenkrankenhaus in Braunschweig (1764-1780). Die Herzöge von Braunschweig trugen im 18. Jahrhundert nicht nur eine vorzügliche Gemäldesammlung zusammen, sondern ließen auch eines der ersten und vorbildlichsten Krankenhäuser der Aufklärung errichten. Interessant ist in diesem Zusammenhang, daß der Baumeister der Braunschweiger Krankenanstalt, Ernst Wilhelm Horn (1732/33-1812), der Vater des später berühmten Berliner Arztes der Charité Ernst Horn (1774-1848) war. Kupferstich um 1790 von Anton August Beck (1718-1787). Stadtarchiv Braunschweig 
von landesherrlichen Obrigkeiten oder reichsunmittelbaren Städten im Zeichen des aufgeklärten Absolutismus, z. B. in Passau (1770-1775), in Stralsund (1782-1784), in Kassel (1783-1785), in Altona (1783-1785), in Karlsruhe (1783-1788) und in Wiesbaden (1785-1789) gegründet oder neu errichtet wurden. Ihre Standorte hatten sie in Haupt- oder Residenzorten, wo man für einen großen Verwaltungsbezirk oder, wie es in den Schweizer Metropolen seit der Biedermeierzeit der Fall war, für den jeweiligen Kanton ein zentrales Allgemeines Krankenhaus einrichtete. Die neuen Anstalten legte man häufig, wie es schon seit dem späten Mittelalter zu beobachten ist, aus hygienischen Grundsätzen in die unmittelbare Nähe von Flüssen und sorgte weitblickend für unbebaute, sonnige und großzügig bemessene Geländeareale.

Eine besondere Ausnahme bildete in der Aufklärungszeit unter den Krankenhausgründungen der Duodezfürsten, Fürstbischöfe und Kommunen das Bürgerspital in Frankfurt am Main (1771-1779), das von dem Arzt Johann Christian Senckenberg (1707-1772) gestiftet wurde. In erster Linie war es der große Wunsch Senckenbergs, mit dieser Hospitalstiftung die Gesundheitsfürsorge seiner Vaterstadt Frankfurt zu verbessern. Aber in der Senckenbergischen Anstalt hatte man die Pfründner immer noch mit berücksichtigt und mit den stationären Kranken unter einem Dach vereinigt. Trotzdem wurde Senckenberg damit, ebenso wie wenig später 1780 der Schweizer Orthopäde Jean-André Venel (1740-1791) aus dem Waadtland durch seine orthopädische Privatklinik in Orbe, zum Vorläufer des privaten Krankenhauswesens, das sich vor allem in der zweiten Hälfte des 19. Jahrhunderts für die fortschrittliche Differenzierung der Medizin in klinische Disziplinen und damit für den Bürger so segensreich erweisen sollte. Ohne solche Fachkliniken hätten sich wahrscheinlich die Augenheilkunde, die Orthopädie und die Kinderheilkunde wesentlich langsamer entwickelt.

Strukturell gliederte man meistens diese neuen, auf heilbare Kranke zugeschnittenen Krankenhäuser der Aufklärung in eine chirurgische, eine innere und manchmal auch in eine geburtshilfliche Abteilung, wenn dafür nicht eigene Gebäude vorhanden oder geplant worden waren. Kranke Frauen und Männer wurden auf streng getrennten Stationen betreut. Die räumliche Abgrenzung bildeten oft die in der Mittelachse gelegenen Verwaltungsräume und die Kapelle, die damit immer noch das zentrale christliche Element bekundete, obwohl sie nach und nach durch die Ausweitung funktionaler Räumlichkeiten (Operationszimmer, Kreißsaal, Rekonvaleszentenzimmer) an den Rand gedrängt werden sollte. 
In der Bevölkerung gewannen diese Allgemeinen Krankenhäuser nur sehr langsam Vertrauen. Die wohlhabenden Schichten zogen es noch lange, ja bis zu Beginn unseres Jahrhunderts vor, sich zu Hause behandeln und pflegen zu lassen. Nicht von ungefähr hatten die damaligen Krankenhäuser den Geruch von Armenanstalten, ja sogar von «Entvölkerungsanstalten». Bezeichnenderweise kam erst 1899 der preußische Medizinalbeamte Adolf Guttstadt zu der Feststellung, daß «die Furcht vor dem Krankenhause den Bestrebungen zur Hilfeleistung nicht mehr hinderlich in den Weg tritt». ${ }^{4}$

Außerdem sorgte man sich seit 1780 um ausreichende hygienische Maßnahmen vor allem in Form von großen Außenfenstern und Oberlichtern über den Flurtüren. Zugleich befahl man zusätzlich die Luft reinigende Verfahren wie die Ausräucherung, wie sie seit 1727 in der Charité üblich waren. Man glaubte, mit solchen Lüftungseinrichtungen den Hospitalismusgefahren, die eine hohe, manchmal über $15 \%$ liegende Sterblichkeit mit sich brachten, vorzubeugen. Gerade die großen Krankenhäuser, wie etwa die Charité in Berlin, waren seit 1800 in geringen zeitlichen Intervallen von den grassierenden «Lazarettfiebern» betroffen. Diese katastrophalen hygienischen Zustände mußten natürlich zum Verruf des Krankenhauses der Biedermeierzeit wesentlich beitragen.

Allgemeine Krankenhäuser der Biedermeierzeit: Das Allgemeine Krankenhaus in Bamberg (1787-1789), Vorbild für zwei Generationen

Dies hatte schon 1789 zu einem neuen baulichen Konzept nach eindeutigen hygienischen Gesichtspunkten geführt, wie es zum erstenmal beispielhaft in Bamberg verwirklicht wurde. Das Bamberger Allgemeine Krankenhaus war von dem Fürstbischof Franz Ludwig von Erthal nach dem Vorbild des Wiener Allgemeinen Krankenhauses (1783-1784) auf Betreiben seines Leibarztes Friedrich Adalbert Marcus (1753-1816) am Rande der Stadt am Flußufer der Regnitz von 1787 bis 1789 in großzügiger Weise gebaut worden. Während in dem Wiener Großkrankenhaus über 2000 Patienten stationär betreut werden konnten, fanden in diesem Hause nur 125 Krankenbetten Platz. Man verfügte über eine chirurgische und eine interne Abteilung. In den kleinen Seitentrakten konnte man auch selbstzahlende, nicht der Armenklasse anheimfallende Patienten aufnehmen (Abb. 8). Eine ärztliche Betreuung für Irre und Schwangere hatte man nicht vorgesehen; dafür sollten im Gegensatz zu dem zwanzig Jahre später vollendeten Fuldaer Landeskrankenhaus (1805-1810) selbständige Anstalten errichtet werden. 


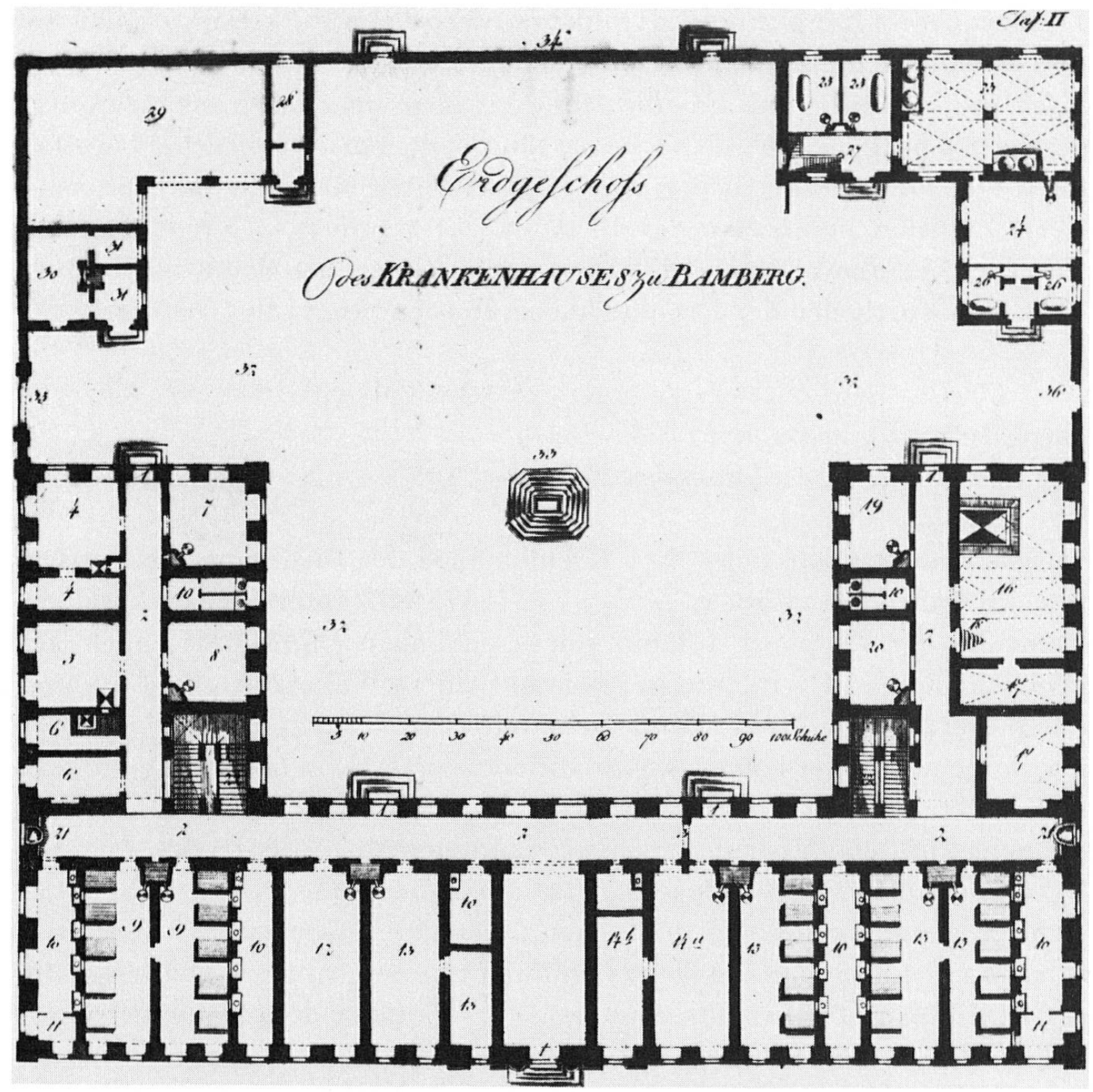

Abb. 8. Grundriß vom Erdgeschoß des Bamberger Krankenhauses. An den beiden Enden des Längstraktes lagen die fünf Betten umfassenden Zimmer der chirurgischen Abteilung. Die Krankenräume waren mit den sogenannten Zwischengängen verbunden, in denen man an den Seiten Toiletten installiert hatte, die mittels eines Schiebers jeder Kranke vom Bett aus ins Zimmer ziehen konnte. Eine ähnliche Anordnung finden wir im ersten Stockwerk, wo über dem Eingang in der Mittelachse die Kapelle liegt. - Zeichenerklärung: 1, 2 = Eingänge und Flure, 3 = Flurabschluß, 4, 5, $6=$ Wohnung des Verwalters, 7, $8=$ Deputationszimmer, $9=$ Krankenzimmer für männliche Patienten, $10=$ Toilettengänge, $11=$ Baderäume, $12=$ Operationsraum, 13 = Zimmer des Wundarztes, 14a = Raum für «clinische Vorlesungen», $14 \mathrm{~b}=$ Apotheke, $16=$ Küche, $17=$ Speisekammern, $18=$ Treppe zum Küchenkeller, $19=$ Treppenaufgänge, 23, 24 = Waschhaus, 25, $26=$ Baderäume, $28=$ Leichenkammer, $29=$ Holzvorrat, $30=$ Wohnung des Knechtes. Aus: Adalbert Friedrich Marcus, Kurze Beschreibung des Allgemeinen Krankenhauses zu Bamberg, Weimar 1797, Tafel II 
Die Bamberger Krankenanstalt bildet insofern einen Markstein, als man mit den sogenannten Zwischengängen besondere sanitäre Einrichtungen für die direkte Beseitigung von Fäkalien und Abfällen aus den Krankenzimmern geschaffen hatte. Diese weitreichende sanitärhygienische Entscheidung galt bis in das ausgehende Biedermeier in ganz Mitteleuropa als nachahmenswert. Zugleich publizierte der Initiator der Bamberger Krankenanstalt Friedrich Adalbert Marcus 1790 ein noch Jahrzehnte diskutiertes Buch «Von den Vortheilen der Krankenhäuser für den Staat». ${ }^{5}$

Das Zürcher Kantonsspital (1839-1841):

Der Operationssaal verdrängt den Betsaal

Das bedeutendste Schweizer Krankenhaus der Biedermeierzeit errichtete die Kantonalbehörde in Zürich von 1837-1842 unter der sachkundigen Beratung des Klinikers Johann Lucas Schönlein (1793-1864) nach den genialen Plänen der Baumeister Gustav Albert Wegmann und Leonhard Zeugheer (Abb. 9). Das 1842 auf einem weiten Areal am Stadtrand in Betrieb genommene Zürcher Kantonsspital differenzierte das in Bamberg spezifisch für ein Krankenhaus geschaffene Konzept. Man schuf, kennzeichnend für die neue klinische Medizin, einen weiträumigen Operationstrakt. Zugleich legte man im Hauptgebäude und im Infektionshaus eine zentrale Warmwasserheizung an, die von dem englischen Ingenieur Perkin entwickelt worden war. Damit hatte man die lästige und nicht immer sauber zu bewerkstelligende Zimmer-Ofenheizung abgelöst. Das Zürcher Krankenhaus wurde bald für zwei Generationen unter dem bedeutenden Chirurgen Heinrich Locher-Zwingli und seinen beiden berühmten Nachfolgern, Theodor Billroth und Edmund Rose, zu einem Mekka der Krankenhausexperten.

Schon der berühmte Wiener Internist Joseph Dietl (1804-1878) hatte 1853 dem Kantonsspital in Zürich, in dem 300 Kranke stationär versorgt werden konnten, das höchste Lob gezollt:

«Abgesondert und doch nicht all zu ferne von der Stadt, auf dem sogenannten Schönhausgute, dem Uetliberge gegenüber, mitten unter grünenden Auen und blumigen Gartenanlagen, frei und erhaben, eine Zierde der Stadt und des Kantones, liegt das KantonalKrankenhaus zu Zürich, den Fremden von Ferne verkündend, daß hier eines der herrlichsten Denkmäler zum Wohle und zur Ehre der leidenden Menschheit aufgeführt wurde! Schwerlich dürfte das Züricher Kantonal-Krankenhaus in Bezug auf Lage und äußere Form von irgendeinem Krankenhause übertroffen werden.» ${ }^{6}$ 


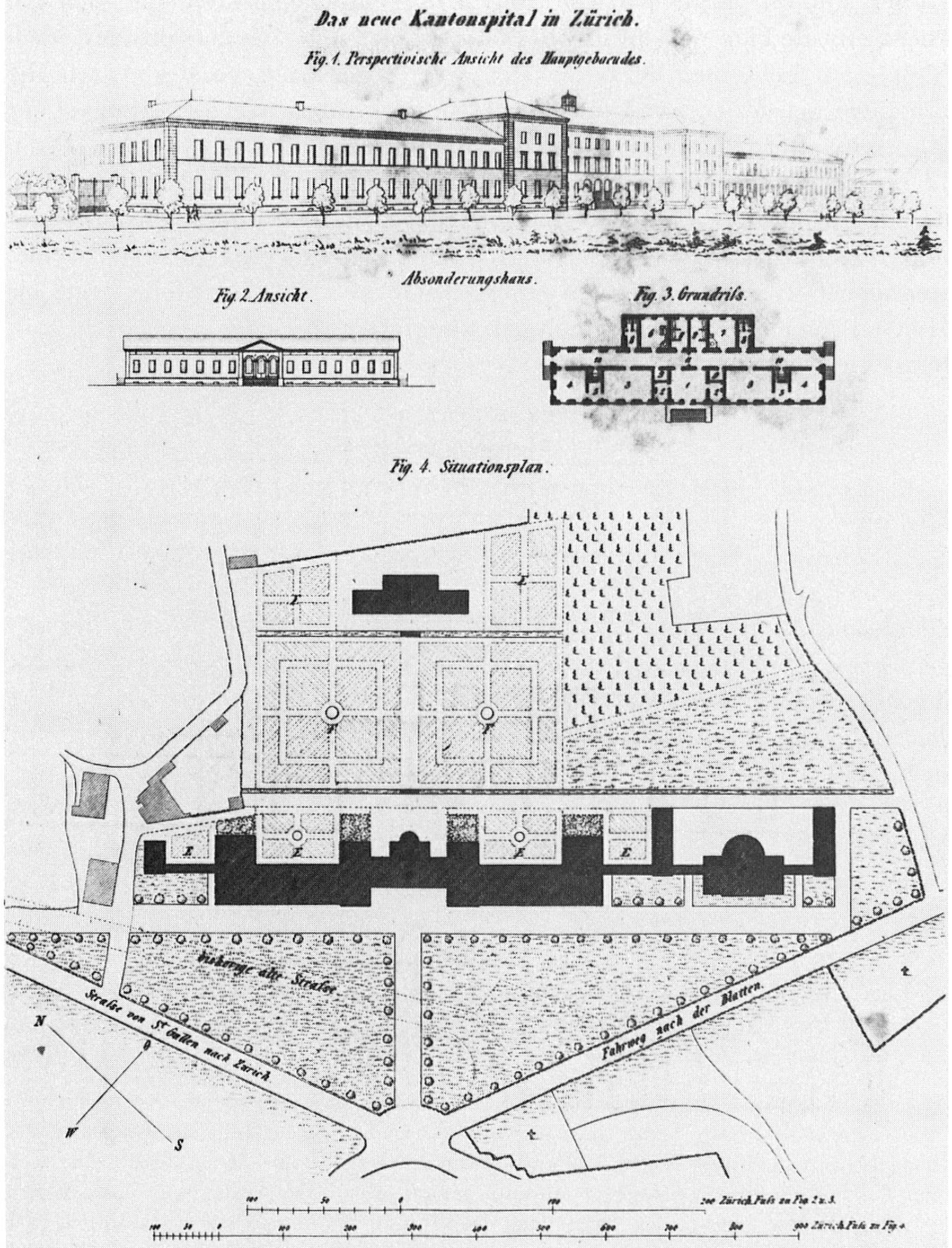

Abb. 9. Das 250 Betten umfassende Kantonsspital in Zürich, das nach den Plänen der beiden Baumeister Gustav Albert Wegmann (1812-1866) und Leonhard Zeugheer (1812-1866) von 1837 bis 1842 errichtet worden ist. Rechts vom Hauptgebäude lag die Anatomie, im hinteren Gartengelände das Infektionshaus. Zeichenerklärung: $\mathrm{Aa}=$ Administrationstrakt, $\mathrm{Ab}=\mathrm{Ab}$ teilung für weibliche Kranke, Ac = Abteilung für männliche Kranke, B = Absonderungshaus, $\mathrm{C}=$ Anatomie, $\mathrm{D}=$ Waschhaus, $\mathrm{E}=$ Garten für die Rekonvaleszenten, $\mathrm{F}=$ Gemüsegärten. Aus: Notizen über das neue Kantonsspital in Zürich. Allgemeine Bauzeitung 8 (1843), S. 355 
Dieser Meinung schlossen sich viele Krankenhausexperten um 1850 an. Nicht nur die klare Gliederung der langgezogenen Gebäudetrakte mit nach Südwesten gelegenen Krankensälen wurde gerühmt, sondern auch die zentrale Lage des halbkreisförmigen Operationsraumes im Mittelteil auf der Nordseite. Der Operateur erhielt sowohl von oben als auch von den Seiten Licht. Rechts und links waren Zimmer angelegt, in denen die Kranken nach der Operation überwacht werden konnten. Sicherlich finden wir auch hier noch einen Raum für geistliche Andachten; aber er ist in erster Linie, wenngleich er noch im ersten Obergeschoß an zentraler Stelle liegt, als Hörsaal gedacht. Als vorbildlich galt ebenfalls damals die separate Erstellung eines Infektionshauses.

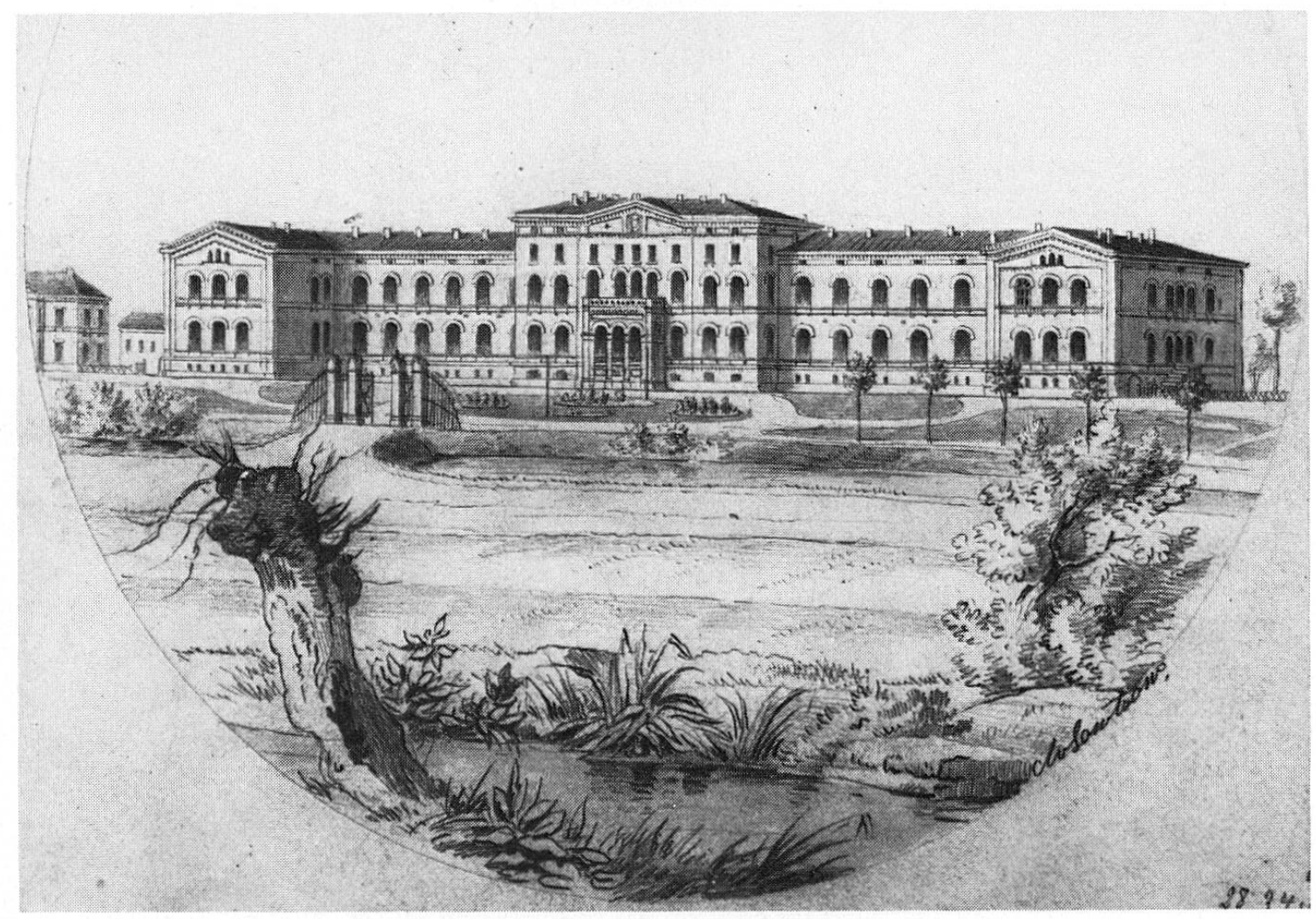

Abb. 10. Blick auf die Hauptfront des 272 Betten umfassenden Allgemeinen Krankenhauses in Bremen (1849-1851). Dieses neuromanische Gebäude wurde aufgrund der Pläne des Baumeisters Alexander Schröder (1806-1877) und des Arztes Daniel Meier (1818-1873) nach dem Zürcher Vorbild in kleinerem Maßstab gebaut. Die Übereinstimmungen zeigen sich besonders in der inneren Strukturierung des Hauses mit dem Operationstrakt in der Mittelachse des Erdgeschosses. Links erkennt man noch die Arztvilla, in der Daniel Meier bis zu seiner vorzeitigen Entlassung 1855 als erster hauptamtlich angestellter Krankenhausarzt der Bremer Bürgerschaft gewohnt hat. Bleistift- und Tuschzeichnung von Alexander von Lowtzow um 1852. Focke-Museum, Bremen 
So wurde das Allgemeine Krankenhaus in Bremen, das heute mit Recht unter Denkmalschutz steht, zusammen mit einer Irrenanstalt und einem Infektionshaus 1851 nach dem Muster des Zürcher Kantonsspitals vollendet (Abb. 10). Maßgeblich war daran neben dem Baumeister Alexander Schröder (1806-1877) der Bremer Arzt Daniel Meier (1812-1873) beteiligt. Er sollte 1855 unter unwürdigen Vorgängen aus seinen Rechten und Pflichten als erster Krankenhausarzt der Freien Hansestadt Bremen entlassen werden und anschließend an der Seite seiner Frau, Louise Aston (1814-1881), einer damals berühmt-berüchtigten Schriftstellerin der Revolution von 1848, ein Vagabundenleben führen ${ }^{7}$. Neben dem Operationsraum auf der Nordseite im Mittelbau lagen auch in Bremen zwei Aufwachkammern. Ebenso wie in Zürich errichtete man ein eigenes Infektionshaus. Noch heute kündet das ehemalige Bremer Krankenhaus von seinem großen schweizerischen Vorbild, das nicht mehr existiert.

Auch der spektakuläre Neubau für die Diakonissenanstalt Bethanien (1845-1847) in Berlin dürfte von Zürich her beeinflußt worden sein, da die Fassadengliederung und Grundrißführung gewisse Übereinstimmungen zeigen (Abb.11). Vermutlich hat der zuständige Baumeister Theodor August Stein (1802-1876) auf einer Studienreise auch die Zürcher Krankenanstalt kennengelernt. Nur verzichtete man im Berliner Bethanien auf einen Operationssaal, da man vorzog, wie vielfach noch üblich, im Krankenzimmer selbst zu operieren. Daß man wahrscheinlich in Berlin das Zürcher Kantonsspital zum Vorbild nahm, mag auch Johann Lucas Schönlein zuzuschreiben sein, der 1841 als Professor an die Berliner Universität berufen und von Friedrich Wilhelm IV., dem großen Förderer dieses Hauses, als sachverständiger ärztlicher Berater hinzugezogen wurde ${ }^{8}$.

In diesem Zusammenhang ist es von erheblichem Interesse, daß man sich bei den Vorbereitungen zum zweiten Neubau des St. Galler Gemeindekrankenhauses (1865-1867), das 1873 mit einem weiteren zusätzlichen Gebäude in den Besitz des 1803 gegründeten Kantons St. Gallen überging, auf einer Studienreise 1864 auch in Augsburg umschaute. Das dort 1859 vollendete Hauptkrankenhaus läßt deutlich, sowohl in seiner äußeren Gestaltung wie in seiner inneren Struktur, das Zürcher Kantonsspital ebenfalls erkennen. Anschließend fuhren der federführende Architekt Johann B. Simon und der ärztliche Initiator des St.Galler Kantonsspitals, Carl Wegelin, noch nach Wien, Berlin, Leipzig, Basel, Neuenburg, Genf und Bern, um schließlich ihre Besichtigungstour im Zürcher Kantonsspital abzuschließen, bevor sie ins heimatliche St. Gallen zurückkehrten. 
Außerdem spielte das St. Galler Kantonsspital neben dem Zürcher in den wechselseitigen Beziehungen zwischen dem Krankenhauswesen der Schweiz und dem des Deutschen Reiches insofern eine entscheidende Rolle, als hier zum erstenmal in der Schweiz Diakonissen aus Kaiserswerth (seit 1849?) tätig waren. Pastor Theodor Fliedner (1800-1864), der 1833 seine Diakonissenanstalt ins Leben gerufen und schon bald aus bescheidenen Anfängen zu einer europäischen Institution entwickelt hatte, sollte bald weitere Diakonissen in Schweizer Krankenhäuser schicken und eigene Anstalten dort gründen (vgl. die Diakonissenhäuser in St-Loup, gegründet 1842; in Bern, gegründet 1836; in Riehen bei Basel, gegründet 1852; in Neumünster bei Zürich, gegründet 1856).

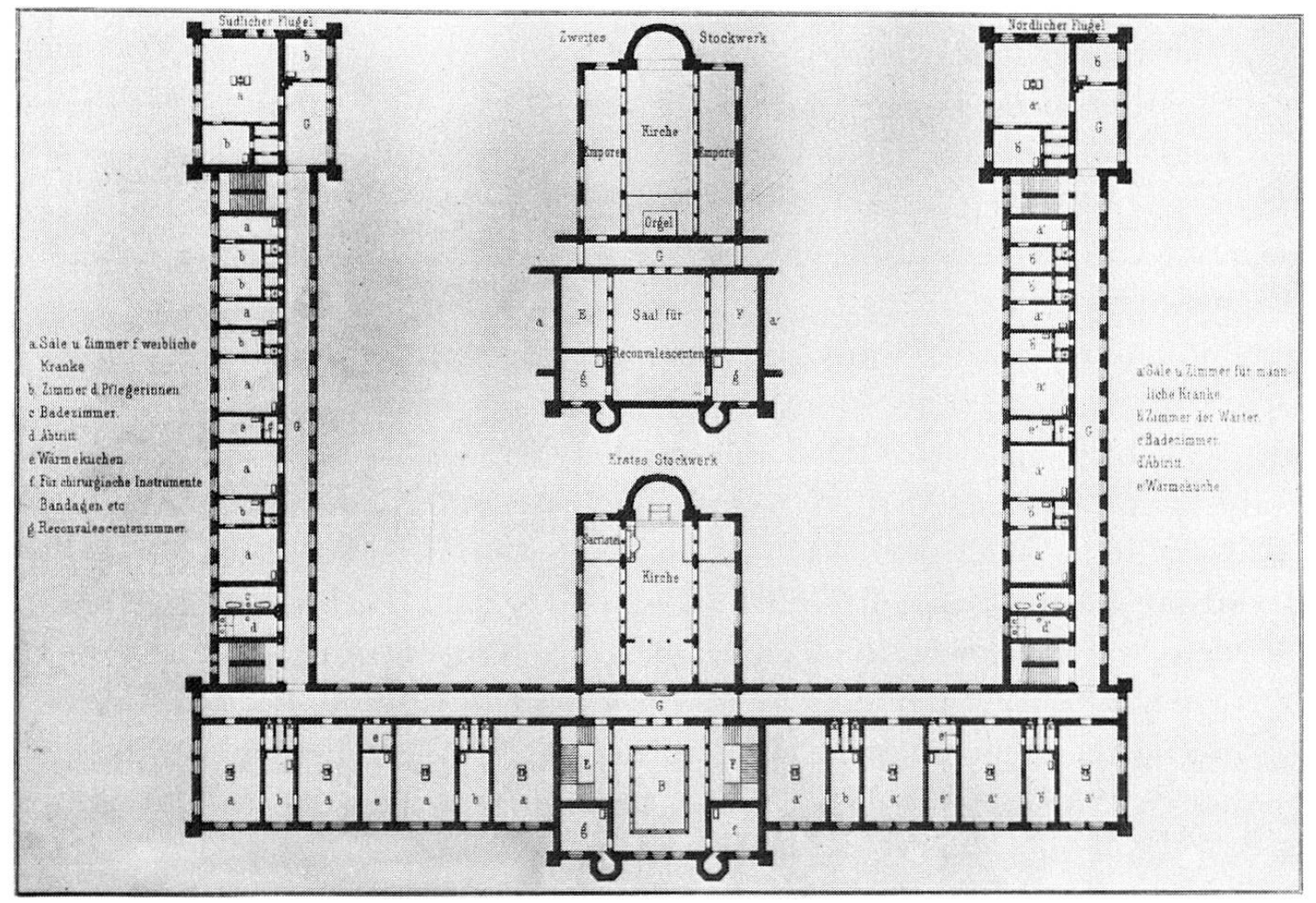

Abb.11. Grundriß vom 1.Stockwerk des Diakonissenkrankenhauses Bethanien in Berlin (1845-1847). Diese evangelische Krankenanstalt wurde unter dem Einfluß des Kaiserswerther Pfarrers Theodor Fliedner (1800-1864) von dem späteren Aachener Architekturprofessor Theodor Stein (1802-1876) errichtet. In der Grundrißführung zeigt Bethanien deutliche Übereinstimmungen mit dem Bamberger und Zürcher Krankenhaus. Nur legte man anstelle des in Zürich auf der Nordostseite ausgebauten Operationstraktes die im byzantinischen Stil ausgestattete Kirche an. Auf einen Operationssaal glaubte man verzichten zu können. Lithographie aus: Theodor Stein, Das Krankenhaus der Diakonissen-Anstalt zu Berlin, Berlin 1850, Tafel 5 
Wenn man auch anfangs den Fliednerschen Diakonissen skeptisch gegenüberstand, so trat doch früh seine in Kaiserswerth praktizierte Krankenpflege durch sachkundig ausgebildete Pflegerinnen aufgrund seines Einflusses auf Florence Nightingale (1820-1910) fast um die ganze Welt ihren Siegeszug an. Neben der schon älteren katholischen Genossenschaft der Barmherzigen Schwestern trugen vor allem die Kaiserswerther Diakonissen ganz entscheidend zur Etablierung der modernen Krankenpflege bei.
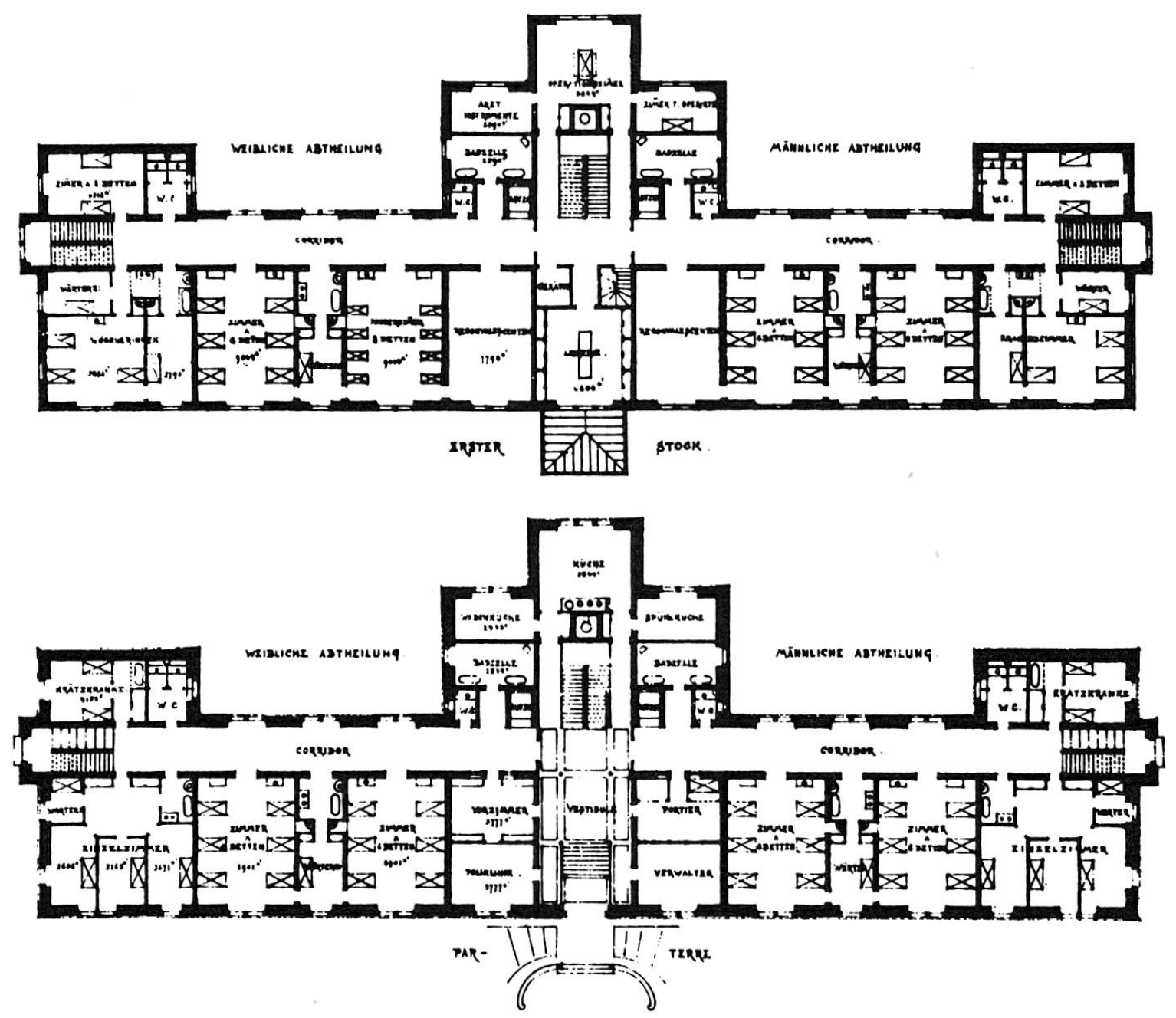

Abb.12. Grundrisse vom sogenannten «Einwohnerspital» in Winterthur, das von 1874 bis 1876 nach den Plänen des Konstanzer Baumeisters Rudolf Friedrich Studer (1855-1926) gebaut wurde. Wie in Zürich hatte man die Krankenzimmer nach Süden ausgerichtet. In den Zwischengängen richtete man Wartezimmer und die Toiletten ein (vgl. auch den Grundriß des Berliner Diakonissen-Krankenhauses Bethanien ). Im ersten Stockwerk lag im Mittelbau auf der Nordseite der Operationstrakt. Aus: A.M.Fehr, 100 Jahre Einwohner- und Kantonsspital Winterthur 1876-1976, Winterthur 1976, S. 16 
Die bald einsetzende Blüte der klinischen Medizin mit ihren seit 1867 immer höheren hygienischen Bedingungen wäre ohne solch geübte, selbstlose Krankenpflegerinnen kaum verstehbar.

Noch eine vorbildliche Krankenanstalt sollte vom Zürcher Kantonsspital wesentlich mitbestimmt werden: das sogenannte Einwohnerspital von Winterthur, das 1876 nach den Plänen des Konstanzer Architekten Rudolf Friedrich Studer (1855-1926), der aus einer berühmten schweizerischen Baumeistersippe stammte, vollendet wurde (Abb.12). Die äußere Fassadenstruktur läßt dies allerdings nicht so sehr deutlich werden - wir finden sie interessanterweise ein Jahrzehnt später im Tettnanger Bezirkskrankenhaus des Stuttgarter Baumeisters Otto Tafel wieder (vgl. Abb. 14) -, um so mehr aber in der inneren Grundrißführung. Selbstverständlich lag auch hier der Operationstrakt im Mittelbau auf der Nordseite. Zugleich finden wir noch den Zwischengang wieder, allerdings nur jeweils einen auf jedem Flügel. Jetzt hatte man in diesen Zwischengängen, wie schon zwanzig Jahre zuvor in Bremen, drei Funktionenzonen eingerichtet: zum seitenständigen Flur ein Badezimmer, in der Mitte zwei Toiletten, die von den angrenzenden Krankenzimmern zugänglich waren, und nach außen ein Wärterzimmer. Die Toiletten wurden 1890, relativ spät, mit einer Wasserspülung versehen; 1904 wurde eine allgemeine elektrische Beleuchtung eingeführt. Vielleicht sollte man auch noch besonders auf das 1895 bezogene Absonderungshaus und auf den 1897 vollendeten Diphtherie-Pavillon hinweisen. Auf diese Weise modifizierte man sinnvoll das in Deutschland bis zum äußersten ausgebaute Pavillonsystem, ohne die bisherigen Bauten aufzugeben ${ }^{9}$. In Hamburg-Eppendorf hatte man ja von 1884 bis 1888 nicht weniger als über 70 Einzelgebäude errichtet; und selbst noch beim 1906 vollendeten RudolfVirchow-Krankenhaus in Berlin entfaltete man eine ganze Stadtlandschaft (vgl. Abb.13).

Eine alternative Lösung zu den Krankenhäusern von Bamberg und Zürich: Das Allgemeine Krankenhaus in München (1808-1813)

Noch ein anderes bauliches Konzept muß hier erwähnt werden, weil es bis heute den Krankenhausbau beeinflußt hat: die Anlage der sanitärtechnischen Räume in kleinen Kammern unmittelbar vor die 10-12 Betten umfassenden Krankenzimmer. Diese sinnvolle Einrichtung von Vorkammern geht auf den Münchner Arzt Franz Xaver Häberl (1759-1846) zurück, 
der in dem von ihm betreuten Spital der Barmherzigen Brüder in München nicht nur Wasserspülklosetts 1796 installierte, außerdem für den seit 1808 begonnenen Neubau des Allgemeinen Krankenhauses München die entscheidenden Pläne lieferte. Seine sanitärtechnischen Ideen und baulichen Vorschläge wurden später vielfach übernommen (Hamburg, 1821-1823; Rotterdam, 1838-1841). Für die Schweizer Krankenhäuser spielte diese funktionelle und sanitärtechnische Gestaltung allerdings nur eine untergeordnete Rolle.

Allgemein kann man sagen, daß etwas später als in Frankreich für den deutschsprachigen Raum um 1810 eine für das Krankenhaus eigene bauliche und strukturelle Entwicklung nach den in Bamberg, Zürich und München geschaffenen Modellen einsetzte, die es seitdem aufgrund hoher Ansprüche an räumliche Funktionalität und an Flexibilität von anderen öffentlichen Institutionen abhebt. Neben gesundheitspolitische, gesellschaftliche und ärztliche Überlegungen traten nun ganz entscheidend die hygienischen Anforderungen, die das Krankenhaus schlechthin in steigendem Maße zu erfüllen hatte.

Sicherlich war es vor allem seit den achtziger Jahren des 18. Jahrhunderts der fachliche Rat der Ärzte, der sich bei der Neuentwicklung von Allgemeinen Krankenhäusern immer erfolgreicher geltend machte. Die medizinische Forschung verlagerte sich, als mit der Französischen Revolution und der darauffolgenden Ära Napoleons in den Pariser Hospitälern die eigentliche Krankenhausmedizin ins Leben gerufen worden war, immer mehr aus der Arztpraxis in diese Institutionen ${ }^{9}$. Denn nirgendwo war man besser in der Lage, den Ansprüchen einer naturwissenschaftlichen Medizin mit neueingeführten diagnostischen Verfahren (Perkussion 1761, Auskultation um 1819, Fiebermessung um 1852) gerecht zu werden und sie in die Praxis umzusetzen, als in diesen Anstalten, in denen die Diagnose und die Therapie von Krankheiten täglich am Bette des Kranken kontrolliert und revidiert werden konnten. Zugleich boten sich die Krankenhäuser als glänzende Unterrichtsstätten für Ärzte an, da das «Bed-side-teaching», das wohl um 1620 im St. Caecilia-Hospital im holländischen Leiden erstmals eingeführt worden war, zunehmend als absolut notwendig angesehen wurde.

Vor allem nach dem in Bamberg, in Fulda und schließlich in Zürich und Bremen zur höchsten Blüte entwickelten Konzept mit den zwischen den Krankenzimmern gelegenen Sanitärzonen zur schnellen, geruchlosen Beseitigung der Fäkalien und mit besonderen Operationsräumen baute man in Mitteleuropa bis in die siebziger Jahre des 19. Jahrhunderts Allgemeine 
Krankenhäuser. Erwähnt seien die teils schon genannten Allgemeinen Krankenhäuser in Stuttgart (1820-1827), in Hannover (1829-1832), in Nürnberg (1839-1845), in Schaffhausen (1843-1846), das Maria-Hilf-Spital in Aachen (1848-1854), das Hauptkrankenhaus in Augsburg (1855-1859), das Einwohnerspital in Winterthur (1874-1876) oder der großzügige Erweiterungstrakt des Kantonsspitals in St.Gallen (1869-1873), die mehr oder weniger diese Grundrißstruktur verwirklichten. Damit folgte man vorwiegend bestimmten hygienischen Hypothesen, die sich an der Geruchlosigkeit der Luft orientierten, und der raschen Ausweitung der Operationstechniken im Bauchraum seit der Einführung der Lachgas-, Äther- und ChloräthylNarkose (1846-1847). So waren bezeichnenderweise seit Zürich und Bremen in den städtischen Krankenhäusern neben dem zentralen Operationsraum zwei Aufwachkammern charakteristisch.

\section{Die Pavillonkrankenhäuser in der zweiten Hälfte des 19. Jahrhunderts}

Trotz umfangreicher sanitärtechnischer Einrichtungen, die vor allem der Reinhaltung der Luft von Gerüchen und sonstigen Ausdünstungen der Kranken dienen sollten, wurden die großen kompakten Krankenhäuser in Basel, Berlin, Hamburg und München von furchterregenden, ärztlich kaum beherrschbaren Wundfieberepidemien heimgesucht. Zur gleichen Zeit grassierte in den geburtshilflichen Kliniken das Kindbettfieber, das eine unendliche Zahl von Frauen hinwegraffte. Ja, damals war für eine Wöchnerin die Gefahr, nach der Entbindung zu sterben, im Krankenhaus um 30\% höher als in ihrer häuslichen Umgebung, so ärmlich sie auch immer gewesen sein mag. Deshalb mußte man nach neuen baulichen und organisatorischen Lösungen suchen. Bei den geburtshilflichen Kliniken versuchte man durch nach dem gleichen Muster eingerichteten Stationen, die im Wechsel halbjährlich belegt werden sollten, um sie die übrige Zeit ausgiebig im leeren Zustand lüften zu können, dem Puerperalfieber entgegenzuwirken. Die 1861 vollendete Hebammenlehranstalt in Hannover galt bis zur Einführung der Antisepsis um 1870 als das große Vorbild. Für die neuen Kinderkliniken galt die Klinik in Basel (1861-1862) als vorbildlich, da sie schon über große, an zwei Seiten mit Außenfenstern versehene Krankensäle und über Sonnenbalkons verfügte. In München folgte man aber nur teilweise beim Neubau des von dem bayrischen Universitätsprofessor August von Hauner (1811-1884) gestifteten Kinderspitales (1880-1881) dem Basler Vorbild, da man - wohl aus 
Kostengründen - auf die Veranden verzichtete. Erst später um 1900 setzte der Pädiater Arthur Schlossmann (1867-1932) solche Terrassen und Balkons für therapeutische Liegekuren in den deutschen Kinderkliniken durch.

In den sechziger Jahren des vorigen Jahrhunderts startete man den Versuch, durch besondere luftige Konstruktionen den Hospitalismus zu bekämpfen. Man entwarf dazu eingeschossige Baracken mit großen Fenstern auf beiden Seiten des Bettensaals und einer Firstventilation. Zusätzlich legte man Ventilationsmaschinen an und wählte poröse, luftdurchlässige Baumaterialien. Auf dem Gelände der Berliner Charité entstand 1866-1867 in Deutschland die erste winterfeste Baracke für Chirurgie unter dem Eindruck von sogenannten Sommerlazaretten der chirurgischen Abteilungen in Leipzig und Nürnberg. Die unmittelbaren Vorläufer zu diesen Barackenhäusern muß man in den Militärlazaretten des Krimkrieges (1853-1855) und des amerikanischen Unabhängigkeitskrieges (1861-1865) suchen. Zugleich erinnerte man sich auch an die schon 1786 und 1788 veröffentlichten Pläne für ein dezentralisiertes Krankenhauswesen im Pavillonstil, das von der Kommission der Pariser Akademie ausgearbeitet worden war. Die ersten Pavillongebäude nach diesen modernen Gesichtspunkten mit ihren länglich-rechteckigen Bettensälen, die von beiden Seiten durch hohe Fenster belichtet und belüftet werden konnten, baute der französische Architekt François Viel für das Hôpital de la Pitié (1802). Vorbildlich wurde aber dann das Pavillonkrankenhaus Lariboisière (1848-1854) in Paris, das bis heute in seiner ursprünglichen Form als Krankenhaus dient.

Spätestens mit der Entwicklung der Antisepsis seit 1867 und der Asepsis um 1886 war man endlich in der Lage, ausreichende desinfizierende Maßnahmen in jedem Gebäude durchzuführen. Die auch damals noch immer geforderte hohe Luftzirkulation im Krankensaal zur Vermeidung von schlechter Luft mit ihren vermeintlichen ansteckenden «Kontagien» hatte vor dem Hintergrund der sich nun etablierenden Bakteriologie ihre eigentliche Berechtigung verloren. Der geniale Bakteriologe Robert Koch (1843-1910) hatte schon 1878 in seinem bahnbrechenden Buch «Über die Aetiologie der Wundinfectionskrankheiten» den Beweis geführt, daß durch die «Kontaktinfektion» der untersuchenden Hände der Ärzte und der unsauberen Verbandmaterialien die gefürchteten Wundfieberepidemien hervorgerufen wurden. Dagegen spielte die für die Hospitalepidemien angeschuldigte «Luftinfektion» eine völlig untergeordnete Rolle.

Trotz dieser neuen Erkenntnisse der Bakteriologie baute man vor allem in Deutschland nach den Vorbildern von Leipzig (1869-1870), Berlin 
(1868-1874) und Hamburg-Eppendorf (1884-1888), aber auch in England und Belgien bis zum Beginn des Ersten Weltkrieges große Pavillonkrankenhäuser (Abb.13). Dagegen blieben die Schweizer Krankenhäuser aufgrund ihrer meist großzügigen baulichen Konzeption in weiten Parkanlagen mit ihren langen dreigeschossigen Bettentrakten von dieser Epoche des Pavillonkrankenhauses nur wenig beeinflußt. Ja teilweise hatten sich im Laufe der Jahrzehnte - wie etwa in St. Gallen - dezentralisierte Blockkrankenhäuser entwickelt. Hinzu kommt die Tatsache, daß schon sehr früh in der Schweiz nach der ersten Publikation von Joseph Lister (1827-1910) im Lancet 1867 sich die Antisepsis durchsetzte. Dies war besonders dem Gynäkologen Johann Jakob Bischoff (1841-1892) zu danken, der schon vor 1868 die Desinfektion mit Karbolsäure am Basler Kantonsspital einführte ${ }^{11}$.

Der berühmte Hamburger Internist Heinrich Curschmann (1846-1910) war einer der eifrigsten Verfechter der dezentralisierten Bauweise, die die

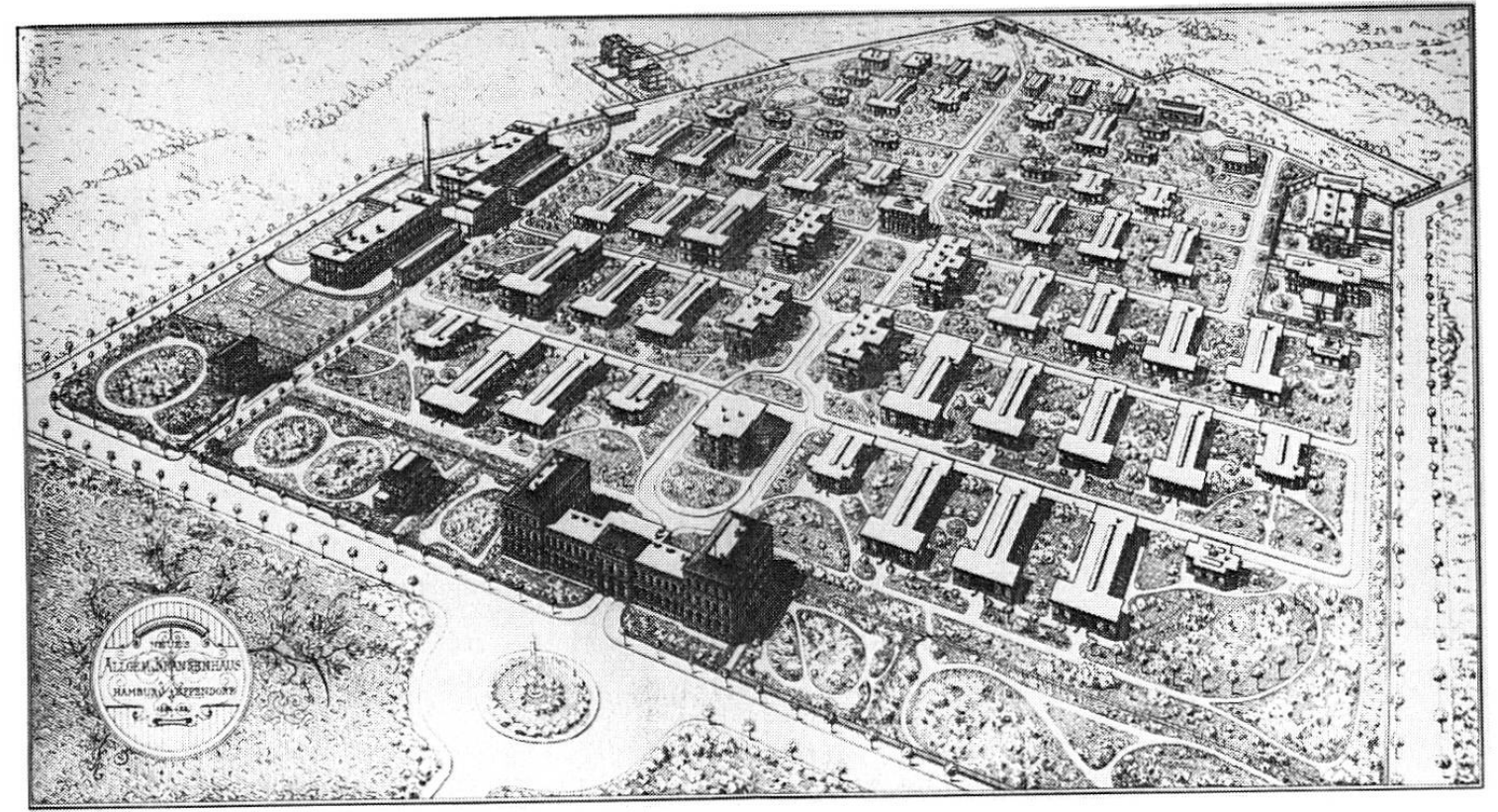

Abb.13. Eine der größten Pavillonanstalten Europas wurde nach den Vorstellungen des späteren Leipziger Internisten Heinrich Curschmann (1846-1910) in Hamburg von 1884 bis 1888 gebaut. Über 70, fast durchweg einstöckige Einzelgebäude errichtete man in mehreren, parallel angeordneten Reihen. In abgewandelter Form wurde dieses Krankenhausmodel vielfach zum Vorbild genommen, z.B. Johns-Hopkins-University Baltimore (1889-1892), Städtisches Krankenhaus Nürnberg (1893-1897), Städtisches Elisabeth-Krankenhaus Aachen (1900-1914). Photolithographie von Strumper. Aus: Hamburg und seine Bauten unter Berücksichtigung der Nachbarstädte Altona und Wandsbeck, Hamburg 1890, S. 234 
Krankenhäuser wie eine Stadtlandschaft mit fünfzig und mehr Einzelgebäuden dezentralisierte (Abb.13). Noch 1906 und 1914 vollendete man große Pavillonanstalten wie das Rudolf-Virchow-Krankenhaus in Berlin oder das städtische Elisabeth-Krankenhaus in Aachen (1900-1914) nach dieser Baustruktur, die den medizin-hygienischen Vorstellungen an sich nicht mehr entsprachen.

Allerdings hatten diese Krankenhausstädte im Grünen den Vorteil, daß man die natürlichen Heilfaktoren in Form von Licht und Luft in den Krankenraum einbeziehen konnte. Schon Curschmann hatte 1888 - auch zur Verteidigung seines Eppendorfer Krankenhaussystems - auf den ganzheitsmedizinischen Aspekt hingewiesen, den man bei den Krankenhäusern berücksichtigen müsse:

«Ist doch das Fernhalten von Infectionsträgern von der Wunde nicht das Einzige, vielmehr ein anderer für den chirurgischen wie für den inneren Kranken gleichgewichtiger Faktor ausschlaggebend, die Sorge für das von der Art des Aufenthalts so wesentlich abhängige allgemeine Körperverhalten.» ${ }^{12}$

In der Schweiz setzten sich dafür die breiten Sonnenterrassen oder Veranden durch, die nach den Vorbildern der Tuberkulosesanatorien in Davos oder Leysin bis 1930 bald bestimmend für das Allgemeine Krankenhaus in Mitteleuropa wurden. So verfügten seit 1907 (Städtisches Krankenhaus Düsseldorf, 1904-1907) auch die neuen städtischen Krankenhäuser in Deutschland über Sonnenbalkone oder zumindest über Veranden, wo man die damals so beliebte Luft- und Lichttherapie durchführen konnte. In der Schweiz bekamen selbst kleine Bezirksspitäler, wie etwa das in Brig, breite Veranden für die Luftkur vorgebaut (Abb. 14).

In diesem Zusammenhang muß nochmals auf das Bezirkskrankenhaus in Tettnang am Bodensee (1884-1886) hingewiesen werden, das nicht nur in seiner genialen kreisförmigen Anlage eine beidseitige Belüftung des Krankenraumes ermöglichte, sondern schon früh zusätzlich breite Veranden zu therapeutischen Zwecken hatte (Abb. 15). Schon der Architekturstil dieses Hauses weist auf Schweizer Vorbilder hin (vgl. das Krankenhaus von Winterthur), wofür auch die überdachten Veranden sprechen, die direkt von einem Sanatorium in Davos übernommen worden sein könnten. Heute ist dieses panoptische Bauprinzip wieder aktuell und hat in dem Oberwalliser Kreisspital Brig (1974-1978), im Kreiskrankenhaus Nordenham (1975-1978) und in den Bettentürmen des Universitätsklinikums Münster (seit 1971 im Bau) spektakuläre Nachahmung in Mitteleuropa gefunden ${ }^{13}$. 
Abb.14. Die Südseite des Kreisspitals in Brig (1907-1909) mit den nach 1916 ausgebauten Seitentrakten und Veranden. Bei der Planung hatte man alle Krankenzimmer auf die Südseite gelegt, so daß in den zwanziger Jahren große Sonnenterrassen ausgebaut werden konnten. Aus: Louis Carlen (Hrsg.), Oberwalliser Kreisspital Brig, 70-jährig und neu erbaut, Brig 1978, S. 26

Man hatte bei den neuen städtischen Krankenhausanlagen in Düsseldorf, Köln (1905-1908), Barmen (1909-1911), München-Schwabing (1909-1911) und Karlsruhe (1908-1912) zu Beginn des 20. Jahrhunderts mit der zwar mehrgeschossigen, doch dezentralisierten Blockbauweise und der betonten Zuordnung des Krankenzimmers zum Parkgelände, zur grünen Natur hin einen tragbaren medizinischen und wirtschaftlichen Kompromiß gefunden. Die einzelnen, auf die klinischen Hauptfächer bezogenen Häuser wurden zwei- bis dreigeschossig angelegt und in eine weiträumige Grünzone gestellt. Teilweise hatte man sie durch unterirdische Gänge verbunden, in denen die Versorgungsleitungen liefen.

In dieser Epoche der Gründerjahre und der Wilhelminischen Zeit von 1871-1918 kam aber die klinische Differenzierung nicht zum Stillstand. Immer mehr Disziplinen, wie etwa die Augenheilkunde, die chirurgische Orthopädie, die Psychiatrie, die Röntgenologie oder die Tuberkulose, bildeten sich als selbständige klinische Einheiten heraus. Zugleich entwickelten sich junge theoretische Fachgebiete der Medizin wie die Hygiene, die Bakteriologie oder die chemische Physiologie zu unverzichtbaren Bestandteilen von Lehre, Forschung und klinischer Praxis. Die Kinderheilkunde wurde zwar lange noch offiziell stiefmütterlich behandelt, aber doch zu Beginn des 20. Jahrhunderts ebenso wie die Augenheilkunde als selbständiges Fach völlig anerkannt, wenn auch die ersten Fachkliniken für Kinderund Augenheilkunde ebenso in der Schweiz wie in Deutschland von Fachärzten oder von Krankenhausvereinen auf eigene Faust ins Leben gerufen und häufig genug aus eigener Tasche (bis zum Ruin der Gründer) finanziert worden waren. Später etablierten sich auch die Dermatologie, die Hals-, Nasen-, Ohrenheilkunde und die Orthopädie zu personell und baulich selbständigen Universitätskliniken.

Abb.15. Das Bezirkskrankenhaus von Tettnang am Bodensee (1884-1885). In dieser Krankenanstalt versuchte der Baumeister Otto Tafel (1838-1914) das Problem der Lüftung nicht durch eine dezentrale Anlageform, sondern durch einen Rundbau zu lösen. Dieses denkmalgeschützte, seit 1976 leerstehende Gebäude geht in seiner Grundrißführung auf utopische Krankenhausentwürfe französischer Revolutionsarchitekten zurück. Die äußere Architektursprache erinnert an Schweizer Vorbilder. - Photographie A. H. Murken, 1979 

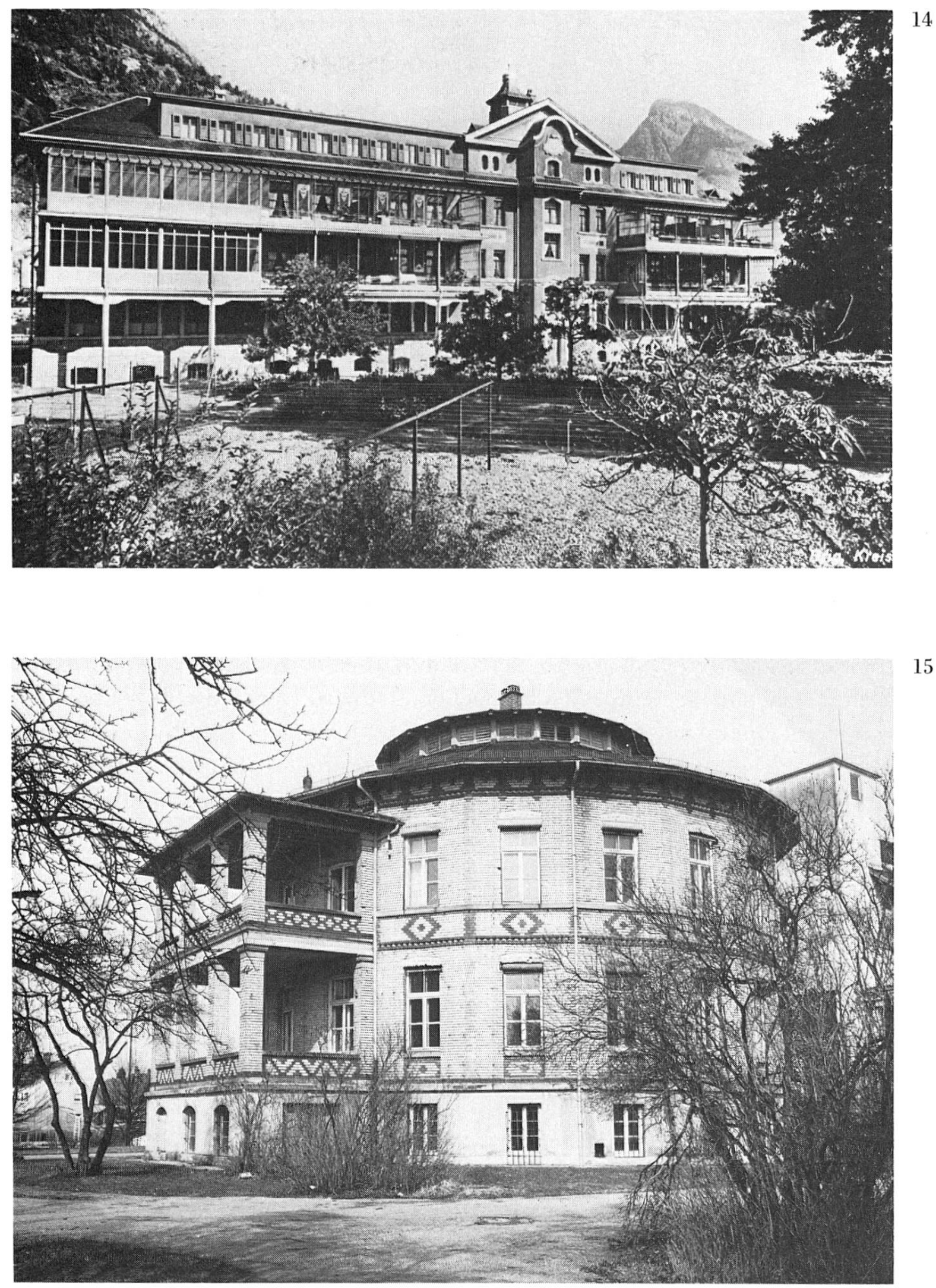
Neue Erkenntnisse in der Hygiene verändern das Krankenhausmilieu seit 1900

Nicht nur die rasche Industrialisierung mit den damit bedingten schlechten Wohnverhältnissen des Kleinbürgertums und des Proletariats veränderten das soziale Verhalten, die Umwelt der Stadtbevölkerung und damit ihre Einstellung zum Krankenhauswesen, sondern auch die früher kaum voraussehbaren Erfolge der praktischen Medizin. Wie kein anderes klinisches Fach erstrahlte die Chirurgie seit 1870, seit der allgemeinen Einführung der Antisepsis, in einem bisher kaum geahnten, spektakulären Glanz. Erkrankungen, wie beispielsweise Blinddarm- oder Gallenblasenentzündungen, die vor der Einführung antiseptischer Methoden in die Operationstechniken mit einem hohen Lebensrisiko verbunden waren, gehörten nach 1880 zunehmend zum erfolgreichen Klinik-Alltag. Die weißen Kittel der Ärzte und Schwestern (seit 1886 zuerst in der chirurgischen Universitätsklinik in Berlin), saubere, mit Desinfektionslösungen gereinigte Operationssäle, speziell konstruierte Krankenhausmöbel und strenge, auf die Hygiene abgestellte Hausregeln ließen die Krankenhäuser in das allgemeine bürgerliche Interesse des Publikums rücken. Die in den Korridoren und Sälen nun überall riechbaren desinfizierenden Chemikalien wurden zu einem positiven Kennzeichen einer keimfreien klinischen Medizin. Die typischen Utensilien aus dem Operationsmilieu - weiße Kittel, Knochensäge, Geburtszange, Mundbinde - avancierten bald zu Statussymbolen des klinisch tätigen Arztes und signalisierten dem Bürger im Krankheitsfall eine fortschrittliche ärztliche Behandlung. Nicht umsonst ließen sich damals so prominente Chirurgen wie Ernst von Bergmann (1836-1907), Theodor Billroth oder später noch Ferdinand Sauerbruch (1875-1951) im Kreise ihrer weißgekittelten, mit Gummihandschuhen (seit 1896) und Mundbinde (seit 1897) versehenen Assistenten und Schwestern gern im Operationssaal wie erfolgreiche, siegesgewohnte Feldherren auf dem Schlachtfeld ablichten.

Die Krankenanstalten und ihre immer mehr Publizität gewinnenden Chefärzte, die bisher, wie der Spitalarzt überhaupt, mehr am Rande des gesellschaftlichen Lebens gestanden hatten, gewannen um 1880 für immer breitere Gesellschaftsschichten an Vertrauen und an Attraktion. Mit gutem Recht errangen damals die hervorragendsten Vertreter der Chirurgenschaft wie der schon genannte Ernst von Bergmann oder Carl Schönborn (1840-1906), Johann Nepomuk Nußbaum (1829-1890) oder Theodor Kocher (1841-1917) das Ansehen von «Halbgöttern in Weiß». Denn nur in 
jenen Anstalten, wo aufgrund strenger hierarchischer Strukturen die Durchführung der noch jungen Desinfektionsmethoden konsequent überwacht wurde, konnte auch das einst so befürchtete Wundfieber besser beherrscht werden. Den Höhepunkt dieser sozialen Entwicklung der klinischen Chirurgie stellte sicherlich Sauerbruch dar, der in Zürich und später in Berlin den preußischen Kasernenhofton in den Operationssaal einführte.

In diesen Jahrzehnten von 1880 bis 1930 entwickelte man als Garantie für eine ausreichende Hygiene eine immer kompliziertere Sanitärtechnik, umfangreichere Operationsvorbereitungen und Narkoseverfahren, die chirurgische Eingriffe im häuslichen Milieu nicht mehr ratsam erscheinen ließen. Die Installation von zentralen Dampfheizungen, technisch ausgefeilten Waschhäusern, elektrischen Lichtanlagen (seit ca. 1890), Wasserklosetts und Badeabteilungen waren in den Krankenhäusern des zweiten deutschen Kaiserreiches und der Schweiz fast selbstverständlich. Auch die ersten pflegeleichten Möbel unserer modernen Zeit aus Gußeisen, Glas, Preßholzplatten wurden für das Krankenhaus konstruiert. Wesentlich später, vierzig Jahre nach den gußeisernen Betten, Stühlen und Nachtschränken von Eppendorf, entwickelte man im Bauhaus in Weimar für die Wohnzimmer des Bürgers diese Modelle erfolgreich weiter. Schließlich führte der Kieler Chirurg Gustav Adalbert Neuber (1850-1932) 1886 in den Operationsräumen seiner Privatklinik für Chirurgie Feinfilteranlagen ein. Er konnte über nicht weniger als fünf Operationsräume verfügen, die nach jeder Operation gründlich gereinigt wurden ${ }^{14}$.

Zugleich erzielte man seit 1890 im Bereich der Tuberkulosebekämpfung aufgrund der Naturheilverfahren in den schon erwähnten Schweizer Sanatorien mit ihren breiten Liegeterrassen und Veranden in klimatisch begünstigten Orten ausgezeichnete Erfolge. Daraus entstanden bald generell Terrassenanlagen oder besondere Tuberkulosepavillons (Luftkurhaus des städtischen Krankenhauses Bremen, 1902) für Allgemeine Krankenhäuser, indem man die allgemeinen natürlichen Heilfaktoren aus gesundheits-medizinischen Gründen auch ins Krankenhaus einbezog. Schließlich konnte in den Terrassenkrankenhäusern in Waiblingen (1928-1930), Maulbronn (1930-1932) und Pforzheim (1932-1934), die bis Mitte der dreißiger Jahre vollendet wurden, der Kranke sogar im Winter in seinem Bett ein heilsames Sonnenbad genießen. Therapeutische Vorstellungen bestimmten nun für eine kurze Weile vollständig die baulichen Strukturen des Krankenhauses, so wie eine Generation zuvor die hygienische Hypothese von der Luftinfektion, die sich nicht halten ließ, zum Pavillonsystem geführt hatte. 


\section{Die ersten kompakten Krankenhäuser vor dem Zweiten Weltkrieg}

Schon seit der Jahrhundertwende lag es nahe, die Zunahme der sanitärtechnischen Einrichtungen, biomedizinischen Apparate und die weitere Differenzierung der klinischen Fächer in Subdisziplinen aus wirtschaftlichen und personaltechnischen Gründen durch eine zentralistische Krankenhausplanung aufzufangen. Gleichzeitig bot der inzwischen hohe Stand der Aufzugtechnik die Möglichkeit, die weiten Horizontalwege durch kurze vertikale Verbindungen abzuschaffen. Damit machte man endgültig den Weg für kompakte Hochhauskrankenhäuser frei. Nur dadurch schien es gegeben, den in den dreißiger Jahren stark fühlbar werdenden Bettenmangel auf ökonomische und personaltechnisch vertretbare Weise einzudämmen. Eines der ersten Beispiele dafür lieferte in Mitteleuropa der Baumeister Ernst Kopp mit dem 1934 vollendeten Martin-Luther-Krankenhaus in Berlin. In der Tat schien gerade in dem nun beginnenden Trend zum Großkrankenhaus, der zuvor in den USA zu beobachten ist, die Möglichkeit gegeben zu sein, das ungeheure Detailwissen der ausufernden klinischen Medizin rational ohne großen Zeitaufwand verfügbar zu machen. Allerdings hat man den Schritt zum kompakten Mammutkrankenhaus im Gegensatz zur Bundesrepublik Deutschland in der Schweiz anscheinend wesentlich behutsamer getan und dezentrale Strukturen - wie es der Neubau des Bürgerspitals in Basel von 1939-1945 zeigt - teilweise nach funktionalen und hygienischen Gesichtspunkten erhalten (Abb.16).

Nach dem Zweiten Weltkrieg sollte sich die Form des weiträumigen, langgestreckten Krankenhauses mit nach Süden geöffneten Fassaden nicht durchsetzen. Die Antibiotika-Ära, die mit der Einführung der ersten Sulfonamide in die Krankenhausmedizin 1935 durch Gerhard Domagk (1895-1964) begann, schien zusammen mit einer strengen Asepsis solche von naturheilkundlichen Vorstellungen getragenen Baukonzepte überflüssig zu machen. Außerdem stieg mit der Bevölkerungszunahme in den Städten und mit den Fortschritten der Labormedizin der Raum- und Bettenbedarf in den Krankenhäusern gewaltig an.

Angesichts der beinahe die Grenzen der menschlichen Psyche überschreitenden Fähigkeiten der naturwissenschaftlich orientierten Heilkunde rükken gegenwärtig ganzheitsmedizinische Gesichtspunkte wie um 1890 wieder in den Vordergrund. Das Eingebettetsein des Menschen in die biologischen Bezugspunkte von Leben und Tod und seine extreme psychische Situation im Krankheitsfall verlangt sicherlich mehr ganzheitliche Beachtung, als 


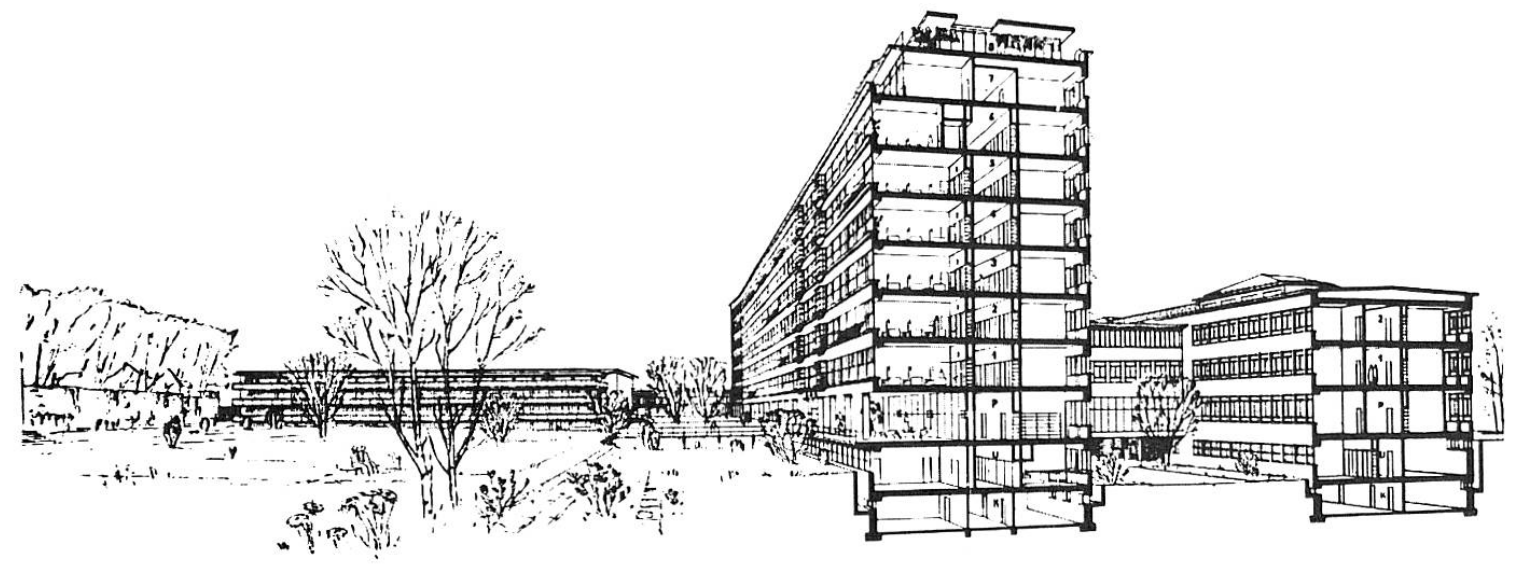

Abb. 16. Schematische Zeichnung vom neuen Bürgerkrankenhaus in Basel (1939-1945). Für die fünfziger und sechziger Jahre unseres Jahrhunderts sollte es - wie schon 1945 in Basel charakteristisch werden, einen hochgeschossigen Bettentrakt (Mitte) mit einem Behandlungsgebäude (rechts) und einem Infektionshaus (links) zu verbinden. Alle Krankenzimmer sind nach Süden gelegen; das oberste Stockwerk (Privatpatienten 1. Klasse) hat zusätzlich große Veranden. Auf dem Dach sind Sonnenterrassen angelegt. Zeichnung aus: E. und P. Vischer, Hermann Baur, Bräuning Leu Dürig: Das neue Bürgerspital in Basel. Schweizerische Bauzeitung 127 (1946), Sonderdruck, S.4

man ihr in den vergangenen zwei Generationen geschenkt hat. Vor allem die seelische und die psychische Ansprache des Patienten, die seit dem Ersten Weltkrieg aus plausiblen ökonomischen und personaltechnischen Gründen wie in der Industrie in ein enges Schema von Funktionsabläufen gedrängt wurde, muß in solch kompliziert strukturierten Krankenhausorganismen oft vernachlässigt werden. Dazu hat natürlich auch der Verlust der religiösen Motivation in der Lebensführung und bei den karitativen Hilfeleistungen beigetragen. Deshalb ist es kaum mehr verwunderlich, daß trotz einer hochleistungsfähigen Krankenhausmedizin die Furcht vor dem Krankenhaus, wie sie im 19. Jahrhundert zu verzeichnen war, seit den letzten zehn Jahren wieder zunimmt.

Wenn heute von der Inhumanität im Krankenhaus gesprochen wird, so sollte man nicht vergessen, daß die heutigen Großkliniken etwa in Aachen, Münster oder Wien wie im Mittelalter die Hospitäler, in der Renaissance die Seuchenlazarette und im 19. Jahrhundert die Allgemeinen Krankenhäuser ein Spiegelbild unserer gesamten Gesellschaft sind. In einem Zeitalter der Weltraumfahrt, das von der Mechanisierung und der Automatisierung bestimmt ist, ist es an sich nur konsequent, gleichfalls die Krankenhäuser wie große Mammutmaschinen zu planen, wie es die französischen Revolutions- 
architekten schon vor 200 Jahren konzipiert haben, in denen die Funktionen der dort tätigen Menschen zwangsläufig immer klarer, enger und zeitlich kürzer schematisiert und kontrolliert werden müssen. Den Höhepunkt dieser Entwicklung stellt sicherlich das neue Klinikum der Technischen Hochschule Aachen dar, das, wie es sich die Krankenhausutopisten der Aufklärung vorstellten, wie eine gigantische Maschine konstruiert ist. Bezeichnend dürfte dafür sein, daß man anstelle eines einzigen Operationsraumes, der im Zürcher Kantonsspital vor 150 Jahren für 300 Kranke völlig ausreichend war, 32 Operationseinheiten für dieses 1400 Betten umfassende klinische Zentrum vorsieht. Ein Traum der naturwissenschaftlich orientierten Medizin ist damit wahr geworden, der nicht unbedingt zu einem Alptraum zu werden braucht.

\section{Anmerkungen}

${ }^{1}$ Rudolf Virchow, Die öffentliche Gesundheitspflege. Medizinische Reform 1 (1848), Nr.9, S. 54: «Allein es darf nicht vorkommen, daß der Staat im konfessionellen Interesse Krankenhäuser errichtet, wie wir es an Bethanien erlebt haben, dessen bloßer Bau deswegen so viel kostet, als das jährliche Armen-Budget der Stadt Berlin beträgt, fast 1/2 Millionen, während es nur einer geringen Zahl von Kranken nützt. Es darf ferner nicht vorkommen, daß Communal-Krankenhäuser im exclusiv particularen Interesse unterhalten werden, daß jeder von der Aufnahme ausgeschlossen wird, der nicht zur Gemeinde gehört und kein Geld hat.»

${ }^{2}$ Vgl. dazu: Marta Meyer-Salzmann, Geschichte der Medizin im Emmental, Sumiswald 1979, S.24ff.; dort ist auch ein Plan abgebildet.

${ }^{3}$ Vgl. Mario Studer, Das amtliche Medizinalwesen im alten Luzern unter besonderer Berücksichtigung der Stadtärzte und ihrer Pflichten, Stans 1958, S.42-43.

${ }^{4}$ Albert Guttstadt, Krankenhaus-Lexikon für das Deutsche Reich. Die Anstaltsfürsorge für Kranke und Gebrechliche und die hygienischen Einrichtungen der Städte im Deutschen Reich am Anfang des zwanzigsten Jahrhunderts. Berlin 1900, S. IX.

${ }^{5}$ Obwohl das Buch von Friedrich Adalbert Marcus, Von den Vortheilen der Krankenhäuser für den Staat, Bamberg und Würzburg 1790, als ein Schlüsselwerk des beginnenden modernen Krankenhauswesens angesehen werden kann, stand er gleichzeitig damit mitten in seiner Zeit. Durch die Veröffentlichungen der Pariser Kommission für den Neuaufbau des Pariser Hôtel-Dieu 1786 und 1788 entspann sich unmittelbar darauf im deutschsprachigen Raum eine angeregte, von Publikationen begleitete Diskussion über das Krankenhaus der Zukunft. An ihr beteiligten sich so unterschiedliche Arztpersönlichkeiten wie der Kurfürstliche Mainzer Leibarzt Christoph Ludwig Hoffmann (1721-1807), der Wiener Medizinalprofessor Maximilian Stoll (1742-1787), der Münchner Stadtarzt Franz Xaver Häberl (1759-1846) oder der Jenaer Medizinprofessor Christoph Wilhelm Hufeland (1762-1836), die sich in der Zeit von 1787 bis 1799 ganz entscheidende Gedanken zum Krankenhauswesen machten. 
${ }^{6}$ Vgl. hierzu besonders Joseph Dietl, Kritische Darstellungen europäischer Krankenhäuser, Wien 1852, Seite 70, wo er ausführlich das St. Galler Kantonsspital, das es damals in dem Sinne noch gar nicht gab (erst ab 1873), behandelte und u. a. schrieb: «Die Krankenpflege wird von zwei Diakonissinen aus Kaiserswerth besorgt ... Die Diakonissinen sollen daher den Spitälern protestantischer Länder das sein, was die Barmherzigen Schwestern den Spitälern katholischer Länder sind. Ob sie das leisten, können wir nach unseren bisherigen Erfahrungen, da das Institut selbst noch zu jung und zu wenig reguliert ist, nicht mit Gewißheit behaupten. Gewiß ist es jedoch, daß schon jetzt zwischen der Krankenpflege der Diakonissinen und der gewöhnlichen Wärtersleute in Bezug auf Ordnung, Reinlichkeit, Gewissenhaftigkeit und Humanität ein unverkennbarer Unterschied zum Vortheile der ersteren sich herausstellt ... Zwei Dinge glauben wir dem verdienstvollen Gründer und Vorsteher der Diakonissinen zur Beherzigung empfehlen zu müssen, erstens: daß er sich mit einem vielerfahrenen und intelligenten Hospitalarzt ins Einvernehmen setze und mit diesem gemeinschaftlich das Institut der Diakonissinen organisiere ... Ferner müssen wir dem verdienstvollen Gründer und Vorsteher der Diakonissen den wohlmeinenden Rat ertheilen, daß er die Kräfte des Instituts nicht all zu sehr zersplittere, oder vielmehr vereinzle, wie dies namentlich im St.Gallener Spital durch Exponierung zweier Diakonissinen der Fall war.»

In diesem Zusammenhang fügte Dietl, die Zürcher Anstalt mit der St.Galler vergleichend, Lob und Tadel hinzu (S.71-72): «Wir haben im Spitale zu St.Gallen denjenigen Grad von Ordnung, Reinlichkeit und Genauigkeit vermißt, den wir im Züricher Kantonalkrankenspitale, und außerdem nur in den Spitälern der barmherzigen Schwestern getroffen, und glauben die Ursache einzig und allein dem so eben erwähnten Umstande zuschreiben zu müssen.»

7 Daniel Meier, Die neue Krankenanstalt in Bremen, 2.Aufl., Bremen 1850, begründet ausführlich, warum er Zürich von allen anderen europäischen Krankenhäusern den Vorzug gab. Das Leben dieses interessanten Arztes und seiner Frau Louise Aston, einer deutschen Pfarrerstochter aus Magdeburg, die früh von einem englischen Fabrikanten geschieden wurde, wäre einer genaueren Untersuchung wert. Louise Aston hat neben Romanen als Augenzeugin die Revolutionswirren von 1848 beschrieben (im Bremer Krankenhaus, wo sie mit ihrem Mann von 1850-1854 wohnte) und kann als eine der intelligentesten europäischen Vorkämpferinnen der Emanzipation der Frau gelten. Wegen dieser revolutionär gesinnten, freigeistigen Frau mußte Meier seinen Chefarztposten in Bremen verlassen. Beide begaben sich bis an ihr Lebensende in Wangen im Allgäu auf eine ständige Wanderschaft durch Rußland, Österreich, die Schweiz und Süddeutschland.

${ }^{8}$ Zum Berliner Bethanien liegt eine Fülle von zeitgenössischer Literatur vor, die vor allem in den Familienzeitschriften des 19. Jahrhunderts ihren Niederschlag gefunden hat. Es ist zugleich eines der am häufigsten dargestellten Krankenhäuser der späten Biedermeierzeit und der anschließenden Restauration.

9 Über das Krankenhaus in Winterthur liegt inzwischen eine glänzende, umfassende Untersuchung vor: A.M.Fehr, 100 Jahre Einwohner- und Kantonsspital Winterthur 1876-1976, Winterthur 1976.

${ }^{10}$ Erwin H.Ackerknecht hat früh auf die Bedeutsamkeit der Pariser Spitäler für die eigentliche naturwissenschaftliche Medizin hingewiesen: Die Pariser Spitäler von 1800 als Ausgangspunkt einer neuen Medizin. Ciba Symp. 7 (1959), S.98-105. In den vergangenen Jahren hat sich besonders Louis S. Greenbaum mit dieser für die Medizin- und Kulturge- 
schichte kaum zu unterschätzenden Epoche befaßt: Health-care and hospital-building in eighteenth-century France. Stud. Voltaire 18th Cent. 152 (1976), S.895-930; Nurses and Doctors in Conflict: Piety and medicine in the Paris Hôtel-Dieu on the eve of the French Revolution. Clio Medica 13 (1979), S.247-267.

${ }^{11}$ Vgl. dazu besonders die interessante Untersuchung von Gwer Reichen, Die chirurgische Abteilung des Bürgerspitals Basel zur Zeit der Antiseptik. Ein Beitrag zur Geschichte der Basler chirurgischen Klinik. Aarau 1949.

${ }^{12}$ Heinrich Curschmann machte diese Bemerkung in einem Vortrag, dessen Thema allein schon Beachtung verdient: Welchen Einfluß hat die heutige Gesundheitslehre, besonders die neue Auffassung des Wesens und der Verbreitung der Infektionskrankheiten auf Bau, Einrichtung und Lage der Krankenhäuser? Vortrag vor der 14. Versammlung des Deutschen Vereins für öffentliche Gesundheitspflege in Frankfurt am Main 1888. Dtsch. Vjschr. öf. Gesd.pfl. 21 (1889), S. 143.

${ }^{13}$ Zum Typ des runden Bettenhauses, das die panoptische Bauweise aus der Gefängnis- und Irrenhausarchitektur ins Krankenhauswesen bringt, vergleiche: Axel Hinrich Murken und Heinz Rodegra, Krankenhausrundbauten in Vergangenheit und Gegenwart. Zur historischen Entwicklung von kreisförmigen Bettenhäusern und panoptisch angelegten Krankensälen. Historia Hospitalium H.13 (1979-1980), S. 197-216.

${ }^{14}$ In der Entwicklung der Antisepsis zur Asepsis nimmt der Kieler Chirurg Adolf Gustav Neuber die entscheidende Rolle ein. Er publizierte auch 1886 über seine aseptischen Methoden (vgl. Neuber 1886). Ein Bild seiner Operationssäle veröffentlichte Albert Landerer in seinem «Handbuch der allgemeinen chirurgischen Pathologie und Therapie», Wien und Leipzig 1890. Leider wird das in den bisherigen Lehrbüchern der Geschichte der Medizin kaum berücksichtigt, obwohl schon 1950 seine Verdienste, die in manchem jene Ernst von Bergmanns übertreffen, ausgezeichnet analysiert wurden: G. E. Konjetzny und E. Heits, Gustav Adolf Neuber und die Asepsis. Eine historische Studie anläßlich des 100. Geburtstages G. A. Neubers am 24. Juni 1950. Stuttgart 1950.

\section{Literaturverzeichnis}

Ackerknecht, Erwin H., Die Pariser Spitäler von 1800 als Ausgangspunkt einer neuen Medizin. Ciba Symposium 7 (1958), S. 98-105.

Bruckner, Albert (Hrsg.), Das Bürgerspital Basel 1260-1946, Basel 1946.

Brunner, Conrad, Über Medizin und Krankenpflege im Mittelalter in Schweizerischen Landen, Zürich 1922.

Buchmann, Kurt, Sankt Gallen als helfende Vaterstadt, St. Gallen 1945.

Carlen, Louis, Oberwalliser Kreisspital Brig, 70-jährig und neu erbaut, Brig 1978.

Curschmann, Heinrich, Welchen Einfluß hat die heutige Gesundheitslehre, besonders die neue Auffassung des Wesens und der Verbreitung der Infektionskrankheiten auf Bau, Einrichtung und Lage der Krankenhäuser? Dtsch. Vjschr. off. Gesd. pfl. 21 (1889), S.139-161.

Deneke, Theodor, Das neue Allgemeine Krankenhaus zu Hamburg-Eppendorf. Unter Mitwirkung von Heinrich Curschmann. Beitrag von H. Schmilinsky. 2. Aufl., Braunschweig 1895.

Dietl, Joseph, Kritische Darstellung europäischer Krankenhäuser. Nach eigenen Reisebeobachtungen. Wien 1852. 
Disselhoff, Julius, Das Diakonissen-Mutterhaus zu Kaiserswerth a.Rh. und seine Tochterhäuser, 2. Aufl., Kaiserswerth 1892.

Fischer, Georg, Chirurgie vor 100 Jahren. Historische Studie über das 18. Jahrhundert aus dem Jahre 1876. Berlin 1876.

Glauser, Fritz (Hrsg.), Kantonales Kreisspital Wolhusen 1972, Wolhusen 1972.

Guttstadt, Albert, Krankenhaus-Lexikon für das Deutsche Reich. Die Anstaltsfürsorge für Kranke und Gebrechliche und die hygienischen Einrichtungen der Städte im Deutschen Reich am Anfang des zwanzigsten Jahrhunderts. Berlin 1900.

Hottinger, A., Das Kinderspital Basel. Historische Studie. Ann. paediat. 199 (1962), S. 1-26.

Hottinger, J. J., und G.von Escher, Das alte und das neue Zürich. Historisch-photographisch beschrieben mit einem Wegweiser durch die Stadt und ihre nächsten Umgebungen. Zürich 1959.

Jetter, Dieter, Geschichte des Hospitals. Westdeutschland von den Anfängen bis 1850. Sudhoffs Arch. Gesch. Med. Naturwiss. Beiheft 5, Wiesbaden 1966.

Dgl., Die ersten Einrichtungen für Arme und Kranke in Westeuropa: Hospitäler aus der Zeit der Merowinger und Karolinger (481-751-987). Sudhoffs Arch. Gesch. Med. Naturwiss. 55 (1971), S. 225-246.

Dgl., Grundzüge der Hospitalgeschichte, Darmstadt 1973.

Kuhn, Oswald F., Krankenhäuser. In: Handbuch der Architektur. Hrsg. Josef Durm, Hermann Ende, Eduard Schmitt und Heinrich Wagner, 4. Teil, 5. Halbband, 1. Heft, Stuttgart 1897; 2. Aufl. Stuttgart 1903.

Lesky, Erna, Das Wiener Allgemeine Krankenhaus. Seine Gründung und Wirkung auf deutsche Spitäler. Clio Medica 2 (1967), S. 23-37.

Marcus, Adalbert Friedrich, Von den Vortheilen der Krankenhäuser für den Staat, Bamberg und Würzburg 1790.

Meyer-Salzmann, Marta, Geschichte der Medizin im Emmental, Sumiswald 1979.

Milt, Bernhard, Geschichte des Zürcher Spitals, Zürich 1951.

Murken, Axel Hinrich, Die Entstehung des Kinderkrankenhauses im 19. Jahrhundert. Med. Mschr. 25 (1971), S.227-235.

Dgl., Aus der Bremer Krankenhausgeschichte: Das Städtische Krankenhaus in Bremen (1849-1851). Ein Architekturmodell des deutschen Krankenhauses um 1850. Historia Hospitalium H.8 (1973), S.49-66.

Dgl., Die Entwicklung und Bedeutung der Anti- und Asepsis für das deutsche Krankenhauswesen von 1867 bis zum Ersten Weltkrieg. Öff. Gesundh.-Wes. 38 (1976), S. 681-696.

Dgl., Das Bild des deutschen Krankenhauses im 19. Jahrhundert, 1. Aufl. Münster 1977; 2. Aufl. Münster 1978.

Dgl., Die Einführung anti- und aseptischer Operationsverfahren im Spiegel der bildenden Kunst von 1875 bis 1912. Med. Mschr. 31 (1977), S. 219-225.

Dgl., Die bauliche Entwicklung des deutschen Allgemeinen Krankenhauses im 19. Jahrhundert, Göttingen 1979.

Dgl., Die Charité in Berlin von 1780 bis 1830. Ein 650 Betten umfassendes Krankenhaus der Biedermeierzeit. Arzt und Krankenhaus 5 (1980), S.20-36.

Murken, Axel Hinrich, und Burkhard Hofmann, Die heilige Elisabeth als Krankenpflegerin. Krankensäle des 15. und 16. Jahrhunderts im Zusammenhang mit Darstellungen der Heiligen Elisabeth von Thüringen. Historia Hospitalium, H. 13 (1979-1980), S. 7-28. 
Neuber, Gustav Adolf, Die aseptische Wundbehandlung in meinen chirurgischen Privathospitälern, Kiel 1886.

Nightingale, Florence, Notes on Hospitals, London 1859.

Dgl., Bemerkungen über Hospitäler. Dtsch. Übersetzung Hugo Senftleben. Memel 1866.

Notizen über das neue Kantonsspital in Zürich. Allg. Bauz. 8 (1843), S. 355-362.

Oppert, Franz, Beschreibung des Hospitals Lariboisière in Paris nebst Bemerkungen über Hospitaleinrichtungen überhaupt. Allg. Bau-Ztg. 23 (1858), S.95-105.

Dgl., Hospitäler und Wohltätigkeitsanstalten, 3. Aufl., Hamburg 1872.

Reichen, Gwer, Die chirurgische Abteilung des Bürgerspitals Basel zur Zeit der Antiseptik. Ein Beitrag zur Geschichte der Basler chirurgischen Klinik. (Veröffentlichungen der Schweizerischen Gesellschaft für Geschichte der Medizin und der Naturwissenschaften, Band 18.) Aarau 1949.

Rennefahrt, Hermann, und Erich Hintzsche: 1354-1954. Sechshundert Jahre Inselspital. Bern 1954.

Rüedi, E., Geschichtliches. In: Das neue Kantonsspital Schaffhausen, Schaffhausen 1954, S. 1-72.

Rüttimann, Beat, Der Balgrist vor 70 Jahren. Zur Geschichte der Orthopädie in der Schweiz. Historia Hospitalium H.14 (1981-1982) (im Druck).

Schimmelbusch, Curt, Anleitung zur aseptischen Wundbehandlung. Vorwort von Ernst von Bergmann. 2. Aufl., Berlin 1893.

Schlossmann, Arthur, Die Düsseldorfer Kranken-, Heil- und Pflegeanstalten, Düsseldorf 1926.

Schrämli, J. J., Das neue Kantonal-Krankenhaus zu Zürich, Zürich 1853.

Steidle, Basilius, Die Regel St.Benedikts, Beuron 1952.

Steinebrunner, Walter Felix, Zwei Zürcher Krankenhausplanungen des 19. Jahrhunderts, ihre ärztlichen Experten, ihre Vorbilder, Diss. med. Zürich 1971.

Steinlin, W., und Carl Wegelin, Über Einrichtung, Bau und Organisation eines St.Gallischen Kantonsspitals, St. Gallen 1861.

Studer, Mario, Das amtliche Medizinalwesen im alten Luzern unter besonderer Berücksichtigung der Stadtärzte und ihrer Pflichten, Diss. med. Basel 1958.

Wegelin, Carl, Geschichte des Ostschweizerischen Säuglingsspitals und des Vereins für Säuglingsfürsorge St.Gallen. 1909-1958. St. Gallen 1960.

Dgl., Geschichte des Kantonsspitals St. Gallen, St. Gallen 1953.

Wegmann, Gustav Albert, und Leonhard Zeugheer, Über die Erbauung eines neuen Krankenhauses für den Kanton Zürich, Zürich 1836. 


\section{Summary}

The hospitals exclusively raised for curable patients developed in the 18th century parallel to the traditional hospitals of the Middle Ages for incurables and old men. At the beginning of the 18th century the new erected hospitals in Bern (Inselspital 1722) or in Berlin (Charite 1727) assembled those curable and incurable patients or persons who were beneficiaries. By this time houses of pestilence partly became transformed into hospitals for curables only (St.Gallen, Hamburg, Leipzig).

Later in the end of the 18th century there arose the first new buildings for curables, so in Braunschweig (1780) or Bamberg (1789). In 1842 the Kantonsspital in Zürich has been erected with a great operating theatre. For two generations the influence of this hospital in Middle Europe can be seen in the "Allgemeine Krankenhäuser" of Berlin (Bethanien 1847), Bremen (1851), Augsburg (1859), and Winterthur (1876). By 1870 the method of pavilion construction began to spread all over Germany and prevailed for the next 40 years (Berlin "Krankenhaus im Friedrichshain" 1874, Hamburg-Eppendorf 1888, Berlin, Rudolf-Virchow-Krankenhaus 1906). Hygienic ideas about the high risks of infection by air set the standards for these pavilion hospitals. After World War I, the pavilion system for hospitals was given up. High story hospitals became determinative in the middle of Europe (MartinLuther-Krankenhaus Berlin 1932, Bürgerspital Basel 1945).

Prof. Dr. med. habil. Axel Hinrich Murken

Abteilung für Geschichte der Medizin

der Rheinisch-Westfälischen Technischen Hochschule Aachen

Kullenhofstraße 50

D-5100 Aachen 